ESAIM: M2AN 48 (2014) 1117-1145

DOI: $10.1051 / \mathrm{m} 2 \mathrm{an} / 2013134$
ESAIM: Mathematical Modelling and Numerical Analysis

www.esaim-m2an.org

\title{
ANISOTROPIC MESH REFINEMENT IN POLYHEDRAL DOMAINS: ERROR ESTIMATES WITH DATA IN $L^{2}(\Omega)$
}

\author{
Thomas Apel ${ }^{1}$, Ariel L. Lombardi ${ }^{2}$ And Max Winkler ${ }^{1}$
}

\begin{abstract}
The paper is concerned with the finite element solution of the Poisson equation with homogeneous Dirichlet boundary condition in a three-dimensional domain. Anisotropic, graded meshes from a former paper are reused for dealing with the singular behaviour of the solution in the vicinity of the non-smooth parts of the boundary. The discretization error is analyzed for the piecewise linear approximation in the $H^{1}(\Omega)$ - and $L^{2}(\Omega)$-norms by using a new quasi-interpolation operator. This new interpolant is introduced in order to prove the estimates for $L^{2}(\Omega)$-data in the differential equation which is not possible for the standard nodal interpolant. These new estimates allow for the extension of certain error estimates for optimal control problems with elliptic partial differential equations and for a simpler proof of the discrete compactness property for edge elements of any order on this kind of finite element meshes.
\end{abstract}

Mathematics Subject Classification. 65N30.

Received March 12, 2013. Revised October 2, 2013.

Published online July 4, 2014.

\section{INTRODUCTION}

We consider the homogeneous Dirichlet problem for the Laplace equation,

$$
-\Delta u=f \quad \text { in } \Omega, \quad u=0 \quad \text { on } \partial \Omega,
$$

where $\Omega$ is a polyhedral domain. Note that we could consider a more general elliptic equation of second order. But by a linear change of the independent variables the main part of the differential operator could be transformed to the Laplace operator in another polyhedral domain such that it is sufficient to consider the Laplace operator here.

The aim of the paper is to prove the discretization error estimate

$$
\left\|u-u_{h}\right\|_{H^{1}(\Omega)} \leq C h\|f\|_{L^{2}(\Omega)}
$$

Keywords and phrases. Elliptic boundary value problem, edge and vertex singularities, finite element method, anisotropic mesh grading, optimal control problem, discrete compactness property.

1 Institut für Mathematik und Bauinformatik, Universität der Bundeswehr München, Germany. thomas.apel@unibw.de; max.winkler@unibw.de

2 Departamento de Matemática, Universidad de Buenos Aires, and Instituto de Ciencias, Universidad Nacional de General

Sarmiento. Member of CONICET, Argentina. aldoc7@dm.uba.ar 
for the finite element solution $u_{h} \in V_{h}$ which is constructed by using piecewise linear and continuous functions on a family of appropriate finite element meshes $\mathcal{T}_{h}$. Note that we assume here not more than $f \in L^{2}(\Omega)$ such that the $L^{2}$-error estimate

$$
\left\|u-u_{h}\right\|_{L^{2}(\Omega)} \leq C h^{2}\|f\|_{L^{2}(\Omega)}
$$

follows by the Aubin-Nitsche method immediately. The generic constant $C$ may have different values on each occurrence.

If the solution of the boundary value problem (1.1) was in $H^{2}(\Omega)$ then the finite element meshes could be chosen quasi-uniform, and the error estimates (1.2) and (1.3) would be standard. However, if the domain $\Omega$ is non-convex, the solution will in general contain vertex and edge singularities, that means $u \notin H^{2}(\Omega)$. In this case the convergence order is reduced in comparison with (1.2) and (1.3) when quasi-uniform meshes are used. As a remedy, we focus here on a priori anisotropic mesh grading techniques as they were investigated by Apel and Nicaise in [4]. In comparison with isotropic local mesh refinement, the use of anisotropic elements avoids an unnecessary refinement along the edges.

The estimate (1.2) is in general proven by using the Céa lemma (or the best approximation property of the finite element method),

$$
\left\|u-u_{h}\right\|_{H^{1}(\Omega)} \leq C \inf _{v_{h} \in V_{h}}\left\|u-v_{h}\right\|_{H^{1}(\Omega)},
$$

and by proving an interpolation error estimate as an upper bound for the right-hand side of (1.4). The particular difficulty is that when the Lagrange interpolant is used together with anisotropic mesh grading, then the local interpolation error estimate

$$
\left|u-I_{h} u\right|_{W^{1, p}(T)} \leq h_{T}|u|_{W^{2, p}(T)}
$$

does not hold for $p=2$ but only for $p>2$, see [2]. Hence the classical proof of a finite element error estimate via

$$
\left\|u-u_{h}\right\|_{H^{1}(\Omega)} \leq C\left\|u-I_{h} u\right\|_{H^{1}(\Omega)} \leq C\left(\sum_{T \in \mathcal{T}_{h}} h_{T}|u|_{H^{2}(T)}^{2}\right)^{1 / 2}
$$

does not work. This problem was overcome by Apel and Nicaise, [4], by using (1.5) and related estimates in weighted spaces, as well as the Hölder inequality for the prize that $f \in L^{p}(\Omega)$ with $p>2$ has to be assumed in problem (1.1). Hence estimate (1.2) cannot be proved in this way.

For prismatic domains and tensor product type meshes the problem was overcome in $[1,6]$ by proving local estimates for a certain quasi-interpolation operator. This work cannot be easily extended to general polyhedral domains since the orthogonality of certain edges of the elements was used there. The aim of the current paper is to construct a quasi-interpolation operator $D_{h}$ such that an error estimate like

$$
\left\|u-D_{h} u\right\|_{H^{1}(\Omega)} \leq C h\|f\|_{L^{2}(\Omega)}
$$

can be proved for the anisotropic meshes introduced in [4].

Quasi-interpolants were introduced by Clément [14]. The idea is to replace nodal values by certain averaged values such that non-smooth functions can be interpolated. This original idea has been modified by many authors since then. The contribution by Scott and Zhang [31] was most influential to our work.

The plan of the paper is as follows. In Section 2 we introduce notation, recall regularity results for the solution $u$ of (1.1) and describe the finite element discretization. The main results are proved in Section 3. The paper continues with numerical results in Section 4 and ends with two sections where we describe applications which motivated us to improve the approximation result from $\left\|u-u_{h}\right\|_{H^{1}(\Omega)} \leq C h\|f\|_{L^{p}(\Omega)}, p>2$, to $\left\|u-u_{h}\right\|_{H^{1}(\Omega)} \leq$ $C h\|f\|_{L^{2}(\Omega)}$. The first one is a discretization of a distributed optimal control problem with (1.1) as the state equation. The second application consists in a simpler proof of the discrete compactness property for edge elements of any order on this kind of finite element meshes.

We finish this introduction by commenting on related work. The idea to treat singularities due to a nonsmooth boundary by using graded finite element meshes is old. The two-dimensional case was investigated by 
Oganesyan and Rukhovets [26], Babuška [9], Raugel [28], and Schatz and Wahlbin [29]. In three dimensions we can distinguish isotropic mesh grading, see the papers by Apel and Heinrich [3] and Apel, Sändig, and Whiteman [5], and anisotropic mesh grading, see the already mentioned papers $[1,2,6]$ for the special case of prismatic domains, and [4] for general polyhedral domains. This work has been extended by Băcuţă, Nistor, and Zikatanov [12] to higher order finite element approximations where naturally higher regularity of the right-hand side $f$ has to be assumed. Boundary element methods with anisotropic, graded meshes have been considered by von Petersdorff and Stephan [27]. The main alternative to mesh grading is augmenting the finite element space with singular functions, see for example Strang and Fix [33], Blum and Dobrowolski [11], or Assous, Ciarlet Jr., and Segré [8] for various variants. It works well in two dimensions where the coefficient in front of the singular function is constant. In the case of edge singularities this coefficient is a function which can be approximated, see Beagles and Whiteman [10], or it can be treated by Fourier analysis, see Lubuma and Nicaise [22].

\section{Notation, Regularity, Discretization}

It is well known that the solution of the boundary value problem (1.1) contains edge and vertex singularities which are characterized by singular exponents. For each edge $e$, the corresponding leading (smallest) singular exponent $\lambda_{e}$ is simply defined by $\lambda_{e}=\pi / \omega_{e}$ where $\omega_{e}$ is the interior dihedral angle at the edge $e$. For vertices $v$ of $\Omega$, the leading singular exponent $\lambda_{v}>0$ has to be computed via the eigenvalue problem of the LaplaceBeltrami operator on the intersection of $\Omega$ and the unit sphere centered at $v$. Note that $\lambda_{e}>\frac{1}{2}$ and $\lambda_{v}>0$. A vertex $v$ or an edge $e$ will be called singular if $\lambda_{v}<\frac{1}{2}$ or $\lambda_{e}<1$, respectively. We exclude the case that $\frac{1}{2}$ is a singular exponent of any vertex. For a detailed discussion of edge and vertex singularities we refer to [16], Sections 2.5 and 2.6 .

As in [4] we subdivide the domain $\Omega$ into a finite number of disjoint tetrahedral subdomains, subsequently called macro-elements,

$$
\bar{\Omega}=\bigcup_{\ell=1}^{L} \overline{\Lambda_{\ell}} .
$$

We assume that each $\Lambda_{\ell}$ contains at most one singular edge and at most one singular vertex. In the case that $\Lambda_{\ell}$ contains both a singular edge and a singular vertex, that vertex is contained in that edge. Note that the edges of $\Lambda_{\ell}$ are considered to have $O(1)$ length. For $\ell_{1} \neq \ell_{2}$, the closures of the macro-elements $\Lambda_{\ell_{1}}$ and $\Lambda_{\ell_{2}}$ may be disjoint or they intersect defining a coupling face, or a coupling edge, or a coupling node.

For the description of the regularity of the solution $u$ of $(1.1)$, we set $\lambda_{\mathrm{v}}^{(\ell)}=\lambda_{v}$ if the macro-element $\Lambda_{\ell}$ contains the singular vertex $v$ of $\Omega$. If $\Lambda_{\ell}$ does not contain any singular vertex we set $\lambda_{\mathrm{v}}^{(\ell)}=+\infty$. Moreover, we set $\lambda_{\mathrm{e}}^{(\ell)}=\lambda_{e}$ if $\Lambda_{\ell}$ contains the singular edge $e$ of $\Omega$, otherwise we set $\lambda_{\mathrm{e}}^{(\ell)}=+\infty$. Furthermore, we define in each macro-element $\Lambda_{\ell}$ a Cartesian coordinate system $x^{(\ell)}=\left(x_{1}^{(\ell)}, x_{2}^{(\ell)}, x_{3}^{(\ell)}\right)$ such that the singular vertex, if existing, is located in the origin, and the singular edge, if existing, is contained in the $x_{3}^{(\ell)}$-axis. We also introduce by

$$
\begin{aligned}
r^{(\ell)}\left(x^{(\ell)}\right) & :=\left(\left(x_{1}^{(\ell)}\right)^{2}+\left(x_{2}^{(\ell)}\right)^{2}\right)^{1 / 2}, \\
R^{(\ell)}\left(x^{(\ell)}\right) & :=\left(\left(x_{1}^{(\ell)}\right)^{2}+\left(x_{2}^{(\ell)}\right)^{2}+\left(x_{3}^{(\ell)}\right)^{2}\right)^{1 / 2}, \\
\theta^{(\ell)}\left(x^{(\ell)}\right) & :=\frac{r^{(\ell)}\left(x^{(\ell)}\right)}{R^{(\ell)}\left(x^{(\ell)}\right)},
\end{aligned}
$$

the distance to the $x_{3}^{(\ell)}$-axis, the distance to the origin, the angular distance from the $x_{3}^{(\ell)}$-axis, respectively.

For $k \in \mathbb{N}$ and $\beta, \delta \in \mathbb{R}$ we define the weighted Sobolev space

$$
V_{\beta, \delta}^{k, 2}\left(\Lambda_{\ell}\right):=\left\{v \in \mathcal{D}^{\prime}\left(\Lambda_{\ell}\right):\|v\|_{V_{\beta, \delta}^{k, 2}\left(\Lambda_{\ell}\right)}<\infty\right\}
$$


where

$$
\begin{aligned}
\|v\|_{V_{\beta, \delta}^{k, 2}\left(\Lambda_{\ell}\right)}^{2} & :=\sum_{|\alpha| \leq k} \int_{\Lambda_{\ell}}\left|R^{\beta-k+|\alpha|} \theta^{\delta-k+|\alpha|} D^{\alpha} v\right|^{2}, \\
|v|_{V_{\beta, \delta}^{k, 2}\left(\Lambda_{\ell}\right)}^{2} & :=\sum_{|\alpha|=k} \int_{\Lambda_{\ell}}\left|R^{\beta} \theta^{\delta} D^{\alpha} v\right|^{2} .
\end{aligned}
$$

Here, we have used the standard multi-index notation to describe partial derivatives, and we have omitted the index $(\ell)$ in $R$ and $\theta$ for simplicity.

Theorem 2.1 (regularity). The weak solution u of the boundary value problem (1.1) admits the decomposition

$$
u=u_{\mathrm{r}}+u_{\mathrm{s}}
$$

in $\Lambda_{\ell}, \ell=1, \ldots, L$, where $u_{\mathrm{r}} \in H^{2}(\Omega) \cap H_{0}^{1}(\Omega)$ and

$$
\frac{\partial u_{\mathrm{s}}}{\partial x_{i}^{(\ell)}} \in V_{\beta, \delta}^{1,2}\left(\Lambda_{\ell}\right), \quad i=1,2, \quad \frac{\partial u_{\mathrm{s}}}{\partial x_{3}^{(\ell)}} \in V_{\beta, 0}^{1,2}\left(\Lambda_{\ell}\right),
$$

for any $\beta, \delta \geq 0$ satisfying $\beta>\frac{1}{2}-\lambda_{\mathrm{v}}^{(\ell)}$ and $\delta>1-\lambda_{\mathrm{e}}^{(\ell)}$.

Proof. The assertion follows widely from [4], Theorem 2.10 where $u_{r} \in H^{2}\left(\Lambda_{\ell}\right)$ was stated. However, when tracing through the proof in that reference, one easily confirms also the global $H^{2}(\Omega)$-regularity.

In [4] an anisotropic mesh refinement strategy for general polyhedra was introduced. The idea is to further decompose the macro-elements according to one of four possible refinement rules described below. By this technique we obtain a conforming triangulation $\mathcal{T}_{h}$ of $\Omega$, i.e.

$$
\bar{\Omega}=\bigcup_{T \in \mathcal{T}_{h}} \bar{T} .
$$

We also assume that $T \cap \Lambda_{\ell} \neq \emptyset$ implies $T \subset \Lambda_{\ell}$. The four refinement rules, which depend on grading parameters $\mu_{\ell}, \nu_{\ell} \in(0,1]$ (see below), are the following:

Type 1. If $\Lambda_{\ell}$ does neither contain a singular edge nor a singular vertex then $\left.\mathcal{T}_{h}\right|_{\Lambda_{\ell}}$ is assumed to be isotropic and quasi-uniform with element size $h$, see Figure 1, left. The grading parameters are taken as $\mu_{\ell}=1$ and $\nu_{\ell}=1$.

Type 2. If $\Lambda_{\ell}$ contains a singular vertex but no singular edges then $\left.\mathcal{T}_{h}\right|_{\Lambda_{\ell}}$ is isotropic and has a singular vertex refinement, i.e., the mesh is graded towards the singular vertex with a grading parameter $\nu_{\ell} \in(0,1]$. This can be achieved by using a coordinate transformation of the vertices from Type 1, see Figure 1, the second from the left. In this case we set $\mu_{\ell}=1$.

Type 3. If $\Lambda_{\ell}$ contains a singular edge but no singular vertex then $\left.\mathcal{T}_{h}\right|_{\Lambda_{\ell}}$ is anisotropically graded towards the singular edge. The grading parameter is $\mu_{\ell} \in(0,1]$. To this end, we introduce a family $\mathcal{P}_{\ell}$ of planes transversal to the singular edge and containing the opposite one. These planes split the macro element into strips and contain all nodes. In the planes the position of the nodes is achieved by applying a coordinate transformation to a uniform triangulation, see Figure 1, the second from the right. We take $\nu_{\ell}=1$.

Type 4. If $\Lambda_{\ell}$ contains both a singular vertex and a singular edge then $\left.\mathcal{T}_{h}\right|_{\Lambda_{\ell}}$ is graded towards the singular edge with grading parameter $\mu_{\ell} \in(0,1]$ and towards the singular vertex with grading parameter $\nu_{\ell} \in(0,1]$, see Figure 1, right. The mesh is topologically equivalent to the mesh of Type 3 but the planes of $\mathcal{P}_{\ell}$ do not divide the singular edge equidistantly but with a grading towards the singular vertex. 

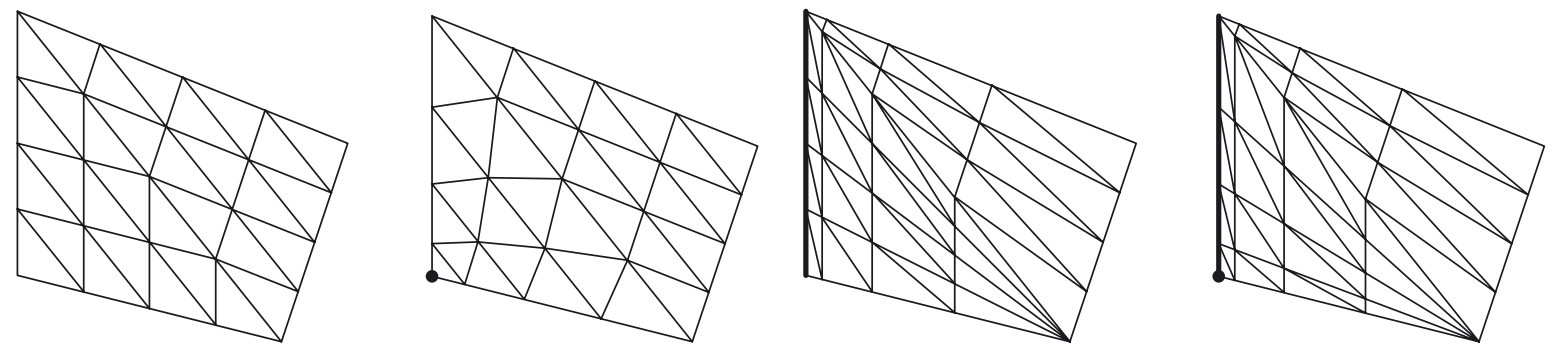

Figure 1. Macro-elements of Type 1, 2, 3 and 4.

We point out that anisotropic elements can appear only in Type 3 and Type 4 , for which $\mathcal{T}_{h}$ contains needle elements near the singular edge and flat elements near the opposite one, see Figure 1. We further observe that if $\Lambda_{\ell}$ is of Type 3 or Type 4 , the elements in $\left.\mathcal{T}_{h}\right|_{\Lambda_{\ell}}$ do not intersect any plane of $\mathcal{P}_{\ell}$.

For each element $T$ we introduce its lengths $h_{1, T}, h_{2, T}, h_{3, T}$ and $h_{T}$ as follows. Let $h_{T}$ be the diameter of $T$. If $T \subset \Lambda_{\ell}$ with $\Lambda_{\ell}$ of Type 1 or Type 2 , then $h_{1, T}=h_{2, T}=h_{3, T}=h_{T}$. If $T \subset \Lambda_{\ell}$ with $\Lambda_{\ell}$ of Type 3 or Type 4 then $h_{3, T}$ is the length of the edge $e_{3, T}$ of $T$ parallel to the singular edge, and $h_{1, T}=h_{2, T}=\frac{1}{2}\left(\left|e_{1, T}\right|+\left|e_{2, T}\right|\right)$ where $e_{1, T}$ and $e_{2, T}$ are the edges of $T$ intersecting $e_{3, T}$ and each one of them is contained in some plane of $\mathcal{P}_{\ell}$.

For the sake of completeness we briefly describe some conditions on the lengths of the elements, and refer to [4] for the details. If $\Lambda_{\ell}$ contains a singular edge, and if $T$ is an element contained in $\Lambda_{\ell}$, define

$$
r_{T}=\inf \left\{r^{(\ell)}\left(x^{(\ell)}\right): x^{(\ell)} \in \Lambda_{\ell}\right\}
$$

and if $\Lambda_{\ell}$ contains a singular vertex

$$
R_{T}=\inf \left\{R^{(\ell)}\left(x^{(\ell)}\right): x^{(\ell)} \in \Lambda_{\ell}\right\} .
$$

Then the length of an arbitrary element $T$ satisfies the following properties. If $\mu_{\ell}<1$ we have

$$
\begin{aligned}
& h_{i, T} \simeq\left\{\begin{array}{c}
h^{1 / \mu_{\ell}} \text { if } r_{T}=0 \\
h r_{T}^{1-\mu_{\ell}} \text { if } r_{T}>0
\end{array} \quad i=1,2\right. \\
& h_{3, T} \leq C\left\{\begin{array}{c}
h^{1 / \nu_{\ell}} \text { if } R_{T} \leq C h^{1 / \nu_{\ell}} \\
h R_{T}^{1-\nu_{\ell}} \text { if } R_{T} \gtrsim h^{1 / \nu_{\ell}}
\end{array}\right. \\
& h_{3, T} \simeq h^{1 / \nu_{\ell}} \text { if } R_{T}=0,
\end{aligned}
$$

and if $\mu_{\ell}=1$ then for $i=1,2,3$

$$
\begin{aligned}
& h_{i, T} \leq C\left\{\begin{array}{cc}
h^{1 / \nu_{\ell}} & \text { if } R_{T} \leq C h^{1 / \nu_{\ell}} \\
h R_{T}^{1-\nu_{\ell}} & \text { if } R_{T} \gtrsim h^{1 / \nu_{\ell}}
\end{array}\right. \\
& h_{i, T} \simeq h^{1 / \nu_{\ell}} \text { if } R_{T}=0 .
\end{aligned}
$$

We also assume that

$$
\mu_{\ell}<1 \Rightarrow \mu_{\ell} \leq \nu_{\ell} .
$$

By classical regularity theory, the solution $u$ of the boundary value problem (1.1) is continuous, see e.g. [16], page 79 , such that the Lagrange interpolant $u_{I}$ with respect to the subdivision $\left\{\Lambda_{\ell}\right\}$ is well defined. We consider the decomposition

$$
u=u_{I}+u_{R} .
$$



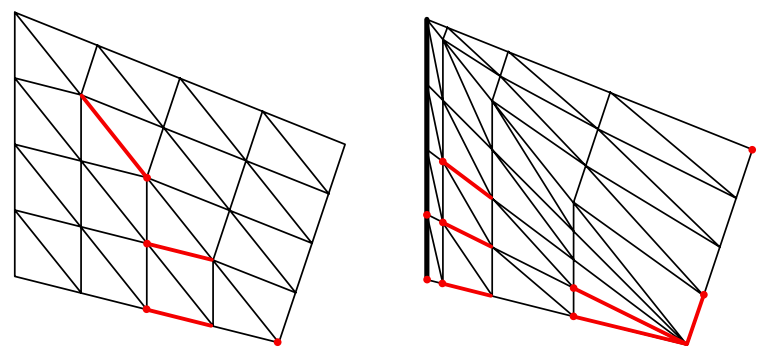

FIGURE 2. Illustration of the edges $\sigma_{n}$.

It follows that the restriction $\left.u_{R}\right|_{\Lambda_{\ell}}$ has the same smoothness properties as $\left.u\right|_{\Lambda_{\ell}}$, see Theorem 2.1. Furthermore, $u_{R}$ vanishes in coupling nodes and on singular edges. We construct now an interpolant $D_{h} u_{R} \in V_{h}$ which also vanishes on these nodes such that $u_{I}+D_{h} u_{R} \in V_{h}$ can be used to estimate the discretization error via (1.4).

To this end, let $\mathcal{N}, \mathcal{N}_{\mathrm{c}}$ and $\mathcal{N}_{\mathrm{s}}$ be the set of all nodes of $\mathcal{T}_{h}$, the set of coupling nodes, and the set of nodes which belong to some singular edge, respectively. The terminal points of the singular edges are included in $\mathcal{N}_{\mathrm{s}}$. For later use, we introduce $\mathcal{N}_{T}:=\mathcal{N} \cap \bar{T}$. The piecewise linear nodal basis on $\mathcal{T}_{h}$ is denoted by $\left\{\phi_{n}\right\}_{n \in \mathcal{N}}$. We associate (as specified below) with each $n \in \mathcal{N} \backslash\left(\mathcal{N}_{\mathrm{c}} \cup \mathcal{N}_{\mathrm{s}}\right)$ an edge $\sigma_{n}$ of some $T \in \mathcal{T}_{h}$ having $n$ as an endpoint. Note that $\left.u\right|_{\sigma_{n}} \in L^{2}\left(\sigma_{n}\right)$ since $u \in H^{s}(\Omega)$ with $s>1$. Hence the operator $D_{h}$ with

$$
D_{h} u=\sum_{n \in \mathcal{N} \backslash\left(\mathcal{N}_{\mathrm{c}} \cup \mathcal{N}_{\mathrm{s}}\right)}\left(\Pi_{\sigma_{n}} u\right)(n) \cdot \phi_{n}(x),
$$

is well defined when $\Pi_{\sigma}: L^{2}(\sigma) \rightarrow P_{1}(\sigma)$ is the $L^{2}(\sigma)$-projection operator onto the space of polynomials of degree less than or equal to one. Note that $D_{h} u$ vanishes on coupling nodes and on singular edges by construction. In order to impose the boundary conditions and to be able to prove interpolation error estimates we need to select the edges $\sigma_{n}$ in an appropriate way, compare the illustration in Figure 2. First, we demand that

- for each node $n \in \mathcal{N} \backslash\left(\mathcal{N}_{\mathrm{c}} \cup \mathcal{N}_{\mathrm{s}}\right), n$ and $\sigma_{n}$ belong to the same macro-elements.

This requires in particular the following restrictions.

- If $n$ lays on a boundary or coupling face, then $\sigma_{n}$ is contained in that face.

- If $n$ lays on a coupling edge, then $\sigma_{n}$ is contained in that coupling edge.

Note that these requirements made the treatment of the coupling nodes via the interpolation on the initial $u_{I}$ necessary. Note further that this construction leads to a preservation of the homogeneous Dirichlet boundary condition.

In order to prove the stability of $D_{h}$ in the anisotropic refinement regions we also require:

- If $n$ is a vertex of a tetrahedron contained in a macro-element $\Lambda_{\ell}$ of Type 3 or Type 4 , then $\sigma_{n}$ is an edge contained on some plane of $\mathcal{P}_{\ell}$.

- In particular, if $T$ contains a node in $\mathcal{N}_{\mathrm{c}} \backslash \mathcal{N}_{\mathrm{s}}$ then $\sigma_{n} \subset \bar{T}$ for all other nodes of $T$.

- If $n_{1}$ and $n_{2}$ belong to a macro-element $\Lambda_{\ell}$ of Type 3 or Type 4 and have the same orthogonal projection onto the $x_{1}^{(\ell)} x_{2}^{(\ell)}$-plane, then the same holds for $\sigma_{n_{1}}$ and $\sigma_{n_{2}}$.

In order to estimate the interpolation error we need to define for each $T \in \mathcal{T}_{h}$ a set $S_{T}$ which should satisfy the following assumptions.

- The set $S_{T}$ is a union of elements of $\mathcal{T}_{h}$ (plus some faces) and in particular $T \subset S_{T}$.

- The set $S_{T}$ is an open connected domain, and as small as possible.

- We have $\sigma_{n} \subset \overline{S_{T}}$ for all nodes $n$ of $T$.

- If $T \subset \Lambda_{\ell}$, then $S_{T} \subset \Lambda_{\ell}$. 

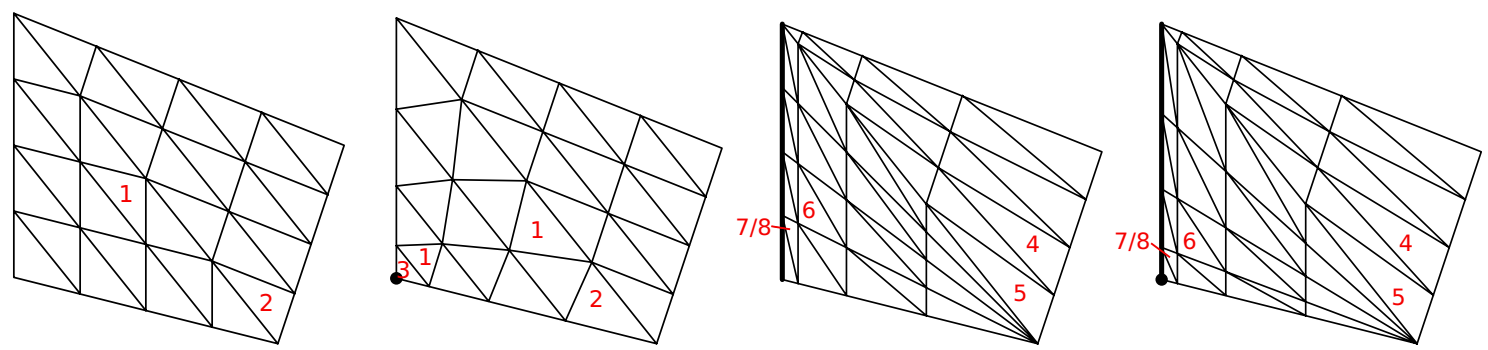

FiguRE 3. Illustration of the cases that have to considered for the interpolation error estimates.

- If $T \subset \Lambda_{\ell}$ with $\Lambda_{\ell}$ of Type 3 or Type 4, then $S_{T}$ lays between two successive planes $\mathcal{P}_{\ell}$. The top and the bottom face of $S_{T}$ are contained in these two planes and the other faces of $S_{T}$ are parallel to the singular edge.

The following properties follow from the definitions of the edges $\sigma_{n}$ and the sets $S_{T}$.

1. Let $T$ be contained in a macro-element $\Lambda_{\ell}$ of Type 3 or Type 4. If $\bar{T}$ intersects two planes $p_{1}$ and $p_{2}$ of $\mathcal{P}_{\ell}$, then $\overline{S_{T}}$ intersects exactly the same planes $p_{1}$ and $p_{2}$.

2. If the node $n, n \notin \mathcal{N}_{\mathrm{c}} \cup \mathcal{N}_{\mathrm{s}}$, belongs to a coupling face, which means that there exist tetrahedra $T_{1} \subset \Lambda_{\ell_{1}}$ and $T_{2} \subset \Lambda_{\ell_{2}}$ with $\ell_{1} \neq \ell_{2}$ and $n \in \mathcal{N}_{T_{1}} \cap \mathcal{N}_{T_{2}}$, then $S_{T_{1}} \cap S_{T_{2}}=\emptyset$ but $\sigma_{n} \subset \overline{S_{T_{1}}} \cap \overline{S_{T_{2}}}$.

3. If $T$ is an isotropic element then all the elements in $S_{T}$ are also isotropic and of size of the same order.

The second point is essential for our proof of the approximation properties. It was the target for which we made the construction as it is.

\section{ERror estimates}

The aim of this section is to derive error estimates for our discretization. They are based on local interpolation error estimates for our interpolant $D_{h}$. For proving these estimates we have to distinguish several cases, see also Figure 3 for an illustration:

1. $T$ is an isotropic element without coupling node, $u$ has full regularity,

2. $T$ is an isotropic element with coupling node, $u$ has full regularity,

3. $T$ is an isotropic element with coupling node, $u$ has reduced regularity,

4. $T$ is an anisotropic flat element without coupling node, $u$ has full regularity,

5. $T$ is an anisotropic flat element with coupling node, $u$ has full regularity,

6. $T$ is an anisotropic needle element without node on the singular edge, $u$ has full regularity,

7. $T$ is an anisotropic needle element with node on the singular edge, $u$ has full regularity.

8. $T$ is an anisotropic needle element with node on the singular edge, $u$ has reduced regularity.

In Lemma 3.1 we present the general approach for the proof of the local interpolation error estimate by considering isotropic elements with and without coupling nodes (cases 1 and 2). We proceed with Lemmas 3.2 where we introduce for isotropic elements how to cope with the weighted norms in the case of reduced regularity (case 3). The interpolated function is only from a weighted Sobolev space but we will see that this even simplifies some parts of the proof.

For anisotropic elements the use of an inverse inequality (as was done in the previous lemmas) has to be avoided; instead we use the structure of the meshes in the macro-elements of Types 3 and Type 4. We start with a stability estimate of $\partial_{3} D_{h} u$ which allows immediately the treatment of anisotropic flat elements (cases 4 and 5) in Lemma 3.4. Then we prove stability estimates for the remaining derivatives and continue with the interpolation error estimates for needle elements. Lemma 3.8 is devoted to cases 6 and 7 , and Lemma 3.10 to case 8 . 
All these local estimates can then be combined to prove the global interpolation error estimate, see Theorem 3.11, and the finite element error estimates, see Corollary 3.13.

Lemma 3.1 (isotropic element, full regularity). If $T$ is an isotropic element then the local interpolation error estimate

$$
\left|u-D_{h} u\right|_{H^{1}(T)} \leq C h_{T}|u|_{H^{2}\left(S_{T}\right)}
$$

holds provided that $u \in H^{2}\left(S_{T}\right)$ and $u(n)=0$ for all $n \in \mathcal{N}_{\mathrm{c}}$.

Proof. Following the explanations in ([31], p. 486), and ([1], p. 1156), an explicit representation of $D_{h} u$ from (2.3) can be given by introducing the unique function $\left.\psi_{n} \in V_{h}\right|_{\sigma_{n}}$ with $\int_{\sigma_{n}} \psi_{n} \phi_{j}=\delta_{n j}$ for all $j \in \mathcal{N}$ such that

$$
\left(\Pi_{\sigma_{n}} u\right)(n)=\int_{\sigma_{n}} u \psi_{n}
$$

and

$$
\left.D_{h} u\right|_{T}=\sum_{n \in \mathcal{N}_{T} \backslash \mathcal{N}_{c}}\left(\int_{\sigma_{n}} u \psi_{n}\right) \cdot \phi_{n} .
$$

Note that

$$
\left\|\psi_{n}\right\|_{L^{\infty}\left(\sigma_{n}\right)}=C\left|\sigma_{n}\right|^{-1}
$$

compare ([1], p. 1157. By some calculation one can even specify that $C=4$.) With (3.3), the inverse inequality

$$
\left|\phi_{n}\right|_{H^{1}(T)} \leq C h_{T}^{-1}|T|^{1 / 2}\left\|\phi_{n}\right\|_{L^{\infty}(T)} \leq C h_{T}^{-1}|T|^{1 / 2},
$$

the trace theorem, see (A.4) with $p=1, q=2$,

$$
\|u\|_{L^{1}\left(\sigma_{n}\right)} \leq C\left|\sigma_{n}\right|\left|S_{T}\right|^{-1 / 2}\left(\|u\|_{L^{2}\left(S_{T}\right)}+h_{T}|u|_{H^{1}\left(S_{T}\right)}+h_{T}^{2}|u|_{H^{2}\left(S_{T}\right)}\right),
$$

and $\left|S_{T}\right| \leq C|T|$ we obtain

$$
\begin{aligned}
\left|D_{h} u\right|_{H^{1}(T)} & \leq C \sum_{n \in \mathcal{N}_{T}}\|u\|_{L^{1}\left(\sigma_{n}\right)}\left\|\psi_{n}\right\|_{L^{\infty}\left(\sigma_{n}\right)}\left|\phi_{n}\right|_{H^{1}(T)} \\
& \leq C h_{T}^{-1}\left(\|u\|_{L^{2}\left(S_{T}\right)}+h_{T}|u|_{H^{1}\left(S_{T}\right)}+h_{T}^{2}|u|_{H^{2}\left(S_{T}\right)}\right) .
\end{aligned}
$$

If $T$ does not contain a node $n \in \mathcal{N}_{\mathrm{c}}$ we find that $D_{h} w=w$ for all $w \in \mathcal{P}_{1}$ such that we get by using the triangle inequality and the stability estimate (3.7)

$$
\begin{aligned}
\left|u-D_{h} u\right|_{H^{1}(T)} & =\left|(u-w)-D_{h}(u-w)\right|_{H^{1}(T)} \quad \forall w \in \mathcal{P}_{1} \\
& \leq|u-w|_{H^{1}(T)}+\left|D_{h}(u-w)\right|_{H^{1}(T)} \\
& \leq C\left(h_{T}^{-1}\|u-w\|_{L^{2}\left(S_{T}\right)}+|u-w|_{H^{1}\left(S_{T}\right)}+h_{T}|u|_{H^{2}\left(S_{T}\right)}\right) .
\end{aligned}
$$

We use now a Deny-Lions type argument (see e.g. [15])

$$
\forall u \in W^{\ell, p}\left(S_{T}\right) \exists w \in P_{\ell-1}: \quad \sum_{j=0}^{\ell} h_{T}^{j}|u-w|_{W^{j, p}\left(S_{T}\right)} \leq C h_{T}^{\ell}|u|_{W^{\ell, p}\left(S_{T}\right)}
$$

and conclude estimate (3.1).

In the case when $\mathcal{N}_{T}$ contains a node $n \in \mathcal{N}_{\mathrm{c}}$ we do not have the property that $D_{h} w=w$ for all $w \in \mathcal{P}_{1}$ but we can use that $u(n)=0$. Let $\sigma_{n}$ be an edge contained in $T$ having $n$ as an endpoint, and let $\phi_{n}$ be the 
Lagrange basis function associated with $n$. (Note that we deal here with nodes $n$ which are not used in the definition of $D_{h}$. Therefore we can assume that $\sigma_{n}$ is local in $\Lambda_{\ell}$.) Consequently, we have with the previous argument that

$$
\left|u-\left(D_{h} u+\left(\Pi_{\sigma_{n}} u\right)(n) \phi_{n}\right)\right|_{H^{1}(T)} \leq C h_{T}|u|_{H^{2}\left(S_{T}\right)} .
$$

Let $I_{T} u$ be the linear Lagrange interpolant of $u$ on $T$. Since $\left.I_{T} u\right|_{\sigma_{n}}$ is linear, we have have $\left(\Pi_{\sigma_{n}} I_{T} u\right)(n)=0$. From this fact and using (3.2)-(3.6) as in the derivation of (3.7) (here with the specific $T$ instead of $S_{T}$ since $\left.\sigma_{n} \subset \bar{T}\right)$, we have

$$
\begin{aligned}
& \left|\left(\Pi_{\sigma_{n}} u\right)(n) \phi_{n}\right|_{H^{1}(T)}=\left|\left(\Pi_{\sigma_{n}}\left(u-I_{T} u\right)\right)(n) \phi_{n}\right|_{H^{1}(T)} \\
& \leq C h_{T}^{-1}\left(\left|u-I_{T} u\right|_{L^{2}(T)}+h_{T}\left|u-I_{T} u\right|_{H^{1}(T)}+h_{T}^{2}|u|_{H^{2}(T)}\right) \\
& \leq C h_{T}|u|_{H^{2}(T)}
\end{aligned}
$$

where we used standard estimates for the Lagrange interpolant in the last step. With (3.9) and the triangle inequality we conclude estimate (3.1) also in this case.

Lemma 3.2 (isotropic element, reduced regularity). If $T$ is an isotropic element with $R_{T}=0$, then the local interpolation error estimate

$$
\left|u-D_{h} u\right|_{H^{1}(T)} \leq C h_{T}^{1-\beta}\|u\|_{V_{\beta, 0}^{2,2}\left(S_{T}\right)}
$$

holds provided that $u \in V_{\beta, 0}^{2,2}\left(S_{T}\right), \beta \in[0,1)$.

Proof. We start as in the proof of Lemma 3.1 but use the sharper trace inequality from Lemma A.1

$$
\|u\|_{L^{1}\left(\sigma_{n}\right)} \leq C\left|\sigma_{n}\right|\left|S_{T}\right|^{-1}\left(\|u\|_{L^{1}\left(S_{T}\right)}+h_{T}|u|_{W^{1,1}\left(S_{T}\right)}+h_{T}^{2}|u|_{W^{2,1}\left(S_{T}\right)}\right) .
$$

With (3.3), (3.4), (3.5), and $\left|S_{T}\right| \leq C|T|$ we obtain

$$
\begin{aligned}
\left|D_{h} u\right|_{H^{1}(T)} & \leq C \sum_{n \in \mathcal{N}_{T}}\|u\|_{L^{1}\left(\sigma_{n}\right)}\left\|\psi_{n}\right\|_{L^{\infty}\left(\sigma_{n}\right)}\left|\phi_{n}\right|_{H^{1}(T)} \\
& \leq C\left|S_{T}\right|^{-1 / 2}\left(h_{T}^{-1}\|u\|_{L^{1}\left(S_{T}\right)}+|u|_{W^{1,1}\left(S_{T}\right)}+h_{T}|u|_{W^{2,1}\left(S_{T}\right)}\right) \\
& \leq C\left(h_{T}^{-1}\|u\|_{L^{2}\left(S_{T}\right)}+|u|_{H^{1}\left(S_{T}\right)}+\left|S_{T}\right|^{-1 / 2} h_{T}|u|_{W^{2,1}\left(S_{T}\right)}\right)
\end{aligned}
$$

and hence via the triangle inequality

$$
\left|u-D_{h} u\right|_{H^{1}(T)} \leq C\left(h_{T}^{-1}\|u\|_{L^{2}\left(S_{T}\right)}+|u|_{H^{1}\left(S_{T}\right)}+\left|S_{T}\right|^{-1 / 2} h_{T}|u|_{W^{2,1}\left(S_{T}\right)}\right) .
$$

Due to the assumed property $R_{T}=0$ we have $0 \leq R(x) \leq h_{T}$ for all $x \in T$, hence $1 \leq h_{T} R^{-1}$, and we obtain

$$
\begin{aligned}
\|u\|_{L^{2}\left(S_{T}\right)} & \leq h_{T}^{2-\beta}\|u\|_{V_{\beta-2,0}^{0,2}\left(S_{T}\right)}, \\
|u|_{H^{1}\left(S_{T}\right)} & \leq h_{T}^{1-\beta}|u|_{V_{\beta-1,0}^{1,2}\left(S_{T}\right)} .
\end{aligned}
$$

To estimate the third term we use the Cauchy-Schwarz inequality and again $R \leq h_{T}$, to obtain for $|\alpha|=2$

$$
\left|D^{\alpha} u\right|_{L^{1}\left(S_{T}\right)} \leq\left\|R^{-\beta}\right\|_{L^{2}\left(S_{T}\right)}\left\|R^{\beta} D^{\alpha} u\right\|_{L^{2}\left(S_{T}\right)} \leq C\left|S_{T}\right|^{1 / 2} h_{T}^{-\beta}|u|_{V_{\beta, 0}^{2,2}\left(S_{T}\right)}
$$

where $\left\|R^{-\beta}\right\|_{L^{2}\left(S_{T}\right)} \leq C\left|S_{T}\right|^{1 / 2} h_{T}^{-\beta}$ is obtained by executing the integration and using that $\beta<\frac{3}{2}$. All these estimates imply estimate (3.10). 
In order to prove interpolation error estimates for the anisotropic elements we derive stability estimates for $D_{h}$ where we avoid the use of the inverse inequality. Let $x_{1}, x_{2}$ and $x_{3}$ be a Cartesian coordinate system with the $x_{3}$-direction parallel to the singular edge of $\Lambda$. We will estimate separately the $L^{2}$-norm of the derivatives of $D_{h} u$.

Moreover, the Deny-Lions type argument (3.8) is not directly applicable for patches of anisotropic elements and requires an appropriate rescaling. For an arbitrary patch $S_{T}$, the coordinate transformation $\left(x_{1}, x_{2}, x_{3}\right)=$ $F\left(\tilde{x}_{1}, \tilde{x}_{2}, \tilde{x}_{3}\right):=\left(h_{1, T} \tilde{x}_{1}, h_{2, T} \tilde{x}_{2}, h_{3, T} \tilde{x}_{3}\right)$ maps a domain $\tilde{S}$ having edge lengths of order one onto $S_{T}$. One further observes that both, the diameter of the largest interior ball in $\tilde{S}$ and the diameter of $\tilde{S}$ itself are of order one. Thus, we conclude from [15] that a polynomial $w \in P_{\ell-1}$ exists (an averaged Taylor polynomial) such that for any multi-index $|\gamma|=m$ the estimate

$$
\left\|\tilde{D}^{\gamma}(\tilde{u}-\tilde{w})\right\|_{W^{\ell-m, p}(\tilde{S})} \leq C\left|\tilde{D}^{\gamma} u\right|_{W^{\ell-m, p}(\tilde{S})}
$$

holds, provided that $0 \leq m \leq \ell$ and $p \in[1, \infty]$. On an arbitrary patch $S_{T}$ we may now apply the transformation onto $\tilde{S}$, where we exploit the property $D^{\alpha} \simeq h_{T}^{-\alpha} \tilde{D}^{\alpha}$, use the Deny-Lions type argument (3.12) on the reference setting and apply the transformation back to $S_{T}$ again which leads to

$$
\begin{aligned}
\sum_{|\alpha|=\ell-m} h_{T}^{\alpha}\left|D^{\alpha}(u-w)\right|_{W^{m, p}\left(S_{T}\right)} & \leq C\left|S_{T}\right|^{1 / p} \sum_{|\gamma|=m} h_{T}^{-\gamma}\left\|\tilde{D}^{\gamma}(\tilde{u}-\tilde{w})\right\|_{W^{\ell-m, p}(\tilde{S})} \\
& \leq C\left|S_{T}\right|^{1 / p} \sum_{|\gamma|=m} h_{T}^{-\gamma}\left|\tilde{D} \tilde{D}^{\gamma} \tilde{u}\right|_{W^{\ell-m, p}(\tilde{S})} \\
& \leq C \sum_{|\alpha|=\ell-m} h_{T}^{\alpha}\left|D^{\alpha} u\right|_{W^{m, p}\left(S_{T}\right)} .
\end{aligned}
$$

Let $T$ be an anisotropic element with the characteristic lengths $h_{1, T}=h_{2, T}$ and $h_{3, T}$. We will not use that $h_{3, T} \geq h_{j, T}, j=1,2$, in the next lemma in order to use this estimate both for the needle and the flat elements.

Lemma 3.3 (stability in direction of the singular edge). For any anisotropic element $T$ the estimate

$$
\left\|\partial_{3} D_{h} u\right\|_{L^{2}(T)} \leq C\left|S_{T}\right|^{-1 / 2} \sum_{|\alpha| \leq 1} h_{T}^{\alpha}\left\|D^{\alpha} \partial_{3} u\right\|_{L^{1}\left(S_{T}\right)}
$$

holds provided that $\partial_{3} u \in W^{1,1}\left(S_{T}\right)$.

Proof. We observe that $T$ has an edge $e_{T}$ parallel to the singular edge, and so, parallel to the $x_{3}$-axis. Since $D_{h} u$ is linear on $T$, we have $\left.\partial_{3} D_{h} u\right|_{T}=\left.\partial_{3} D_{h} u\right|_{e_{T}}$. If $e_{T}$ is contained on the singular edge, then $\left.\partial_{3} D_{h} u\right|_{T}=0$ since $\left.D_{h} u\right|_{e_{T}}=\left.u\right|_{e_{T}}=0$ and we are done. Now, consider the case that $e_{T}$ is not contained in a singular edge and denote its endpoints by $n_{1}$ and $n_{2}$ such that $\left.\partial_{3} \phi_{n_{1}}\right|_{T}=-h_{3, T}^{-1}$ and $\left.\partial_{3} \phi_{n_{2}}\right|_{T}=h_{3, T}^{-1}$. Then we have

$$
\partial_{3} D_{h} u=h_{3, T}^{-1}\left[\int_{\sigma_{n_{2}}} u \psi_{n_{2}}-\int_{\sigma_{n_{1}}} u \psi_{n_{1}}\right]
$$

We observe now that by our assumptions $\sigma_{n_{1}}$ and $\sigma_{n_{2}}$ have the same projection $\sigma_{T}$ into the $x_{1} x_{2}$-plane and hence form two opposite edges of a plane quadrilateral or triangle which is parallel to the $x_{3}$-axis and which we will denote by $F_{T}$. We note further that $\psi_{n_{1}}$ and $\psi_{n_{2}}$ can be considered as the same function $\psi_{T}$ defined on $\sigma_{T}$ and $\left\|\psi_{T}\right\|_{L^{\infty}\left(\sigma_{T}\right)}=C\left|\sigma_{T}\right|^{-1}$. Moreover, the relation $\left|F_{T}\right| \simeq h_{3, T}\left|\sigma_{n}\right|$ holds. With this insight we obtain

$$
\begin{aligned}
\left|\partial_{3} D_{h} u\right| & =h_{3}^{-1}\left|\int_{\sigma_{n_{2}}} u \psi_{n_{2}}-\int_{\sigma_{n_{1}}} u \psi_{n_{1}}\right|=h_{3, T}^{-1}\left|\int_{F_{T}} \partial_{3} u \psi_{T}\right| \\
& \leq C h_{3, T}^{-1}\left|\sigma_{T}\right|^{-1}\left\|\partial_{3} u\right\|_{L^{1}\left(F_{T}\right)} \leq C\left|F_{T}\right|^{-1}\left\|\partial_{3} u\right\|_{L^{1}\left(F_{T}\right)} .
\end{aligned}
$$


We integrate this estimate over $T$, apply the standard trace theorem

$$
\|v\|_{L^{1}\left(F_{T}\right)} \leq C\left|F_{T}\left\|\left.S_{T}\right|^{-1} \sum_{|\alpha| \leq 1} h_{T}^{\alpha}\right\| D^{\alpha} v \|_{L^{1}\left(S_{T}\right)}\right.
$$

and obtain the desired estimate.

We are now prepared to estimate the interpolation error for the flat elements occurring far away from the singular edge in cases 4 and 5 .

Lemma 3.4 (anisotropic flat element, full regularity). If $T$ is an anisotropic flat element $\left(h_{3, T} \leq h_{1, T}=h_{2, T}\right)$ then the local interpolation error estimate

$$
\left|u-D_{h} u\right|_{H^{1}(T)} \leq C \sum_{|\alpha|=1} h_{T}^{\alpha}\left|D^{\alpha} u\right|_{H^{1}\left(S_{T}\right)}
$$

holds provided that $u \in H^{2}\left(S_{T}\right)$.

Proof. The proof for $\partial_{3}\left(u-D_{h} u\right)$ can be done on the basis of Lemma 3.3. Assume for the moment that the element $T$ does not contain a coupling node. Similar to the proof of Lemma 3.1 we obtain for any first-order polynomial $w \in P_{1}$

$$
\left\|\partial_{3}\left(u-D_{h} u\right)\right\|_{L^{2}(T)}=\left\|\partial_{3}(u-w)-\partial_{3} D_{h}(u-w)\right\|_{L^{2}(T)} \leq C \sum_{|\alpha| \leq 1} h_{T}^{\alpha}\left\|D^{\alpha} \partial_{3}(u-w)\right\|_{L^{2}\left(S_{T}\right)} .
$$

Applying now the Deny-Lions type argument (3.13) with $\ell=1$ and $m=0$ leads to the estimate

$$
\left\|\partial_{3}\left(u-D_{h} u\right)\right\|_{L^{2}(T)} \leq C \sum_{|\alpha|=1} h_{T}^{\alpha}\left\|D^{\alpha} \partial_{3} u\right\|_{L^{2}\left(S_{T}\right)} .
$$

Note that the polynomial $w$ can be chosen such that it vanishes in three nodes of $T$. It is completely described by choosing the appropriate value at one endpoint of the edge of $T$ which is parallel to the $x_{3}$-axis. Since a possible coupling node is not an endpoint of this edge, the argument above can also be used in the case of coupling nodes.

For the other directions we can proceed as in the proof of Lemma 3.1. If $T$ contains a coupling node we assumed that $\sigma_{n} \subset \bar{T}$ for all $n \in \mathcal{N}_{T} \backslash \mathcal{N}_{\mathrm{c}}$. Thus, we may apply the trace theorem, see (A.3), which reads in our setting

$$
\|v\|_{L^{1}\left(\sigma_{n}\right)} \leq C\left|\sigma_{n}\left\|\left.T\right|^{-1 / 2} \sum_{|\alpha| \leq 2} h_{T}^{\alpha}\right\| D^{\alpha} v \|_{L^{2}(T)}\right.
$$

instead of (3.6). Then we obtain together with $\left\|\partial_{i} \phi_{n}\right\|_{L^{2}(T)} \leq C h_{i, T}^{-1}|T|^{1 / 2}$ and $\|\psi\|_{L^{\infty}\left(\sigma_{n}\right)} \leq C\left|\sigma_{n}\right|^{-1}$

$$
\begin{aligned}
\left\|\partial_{i} D_{h} v\right\|_{L^{2}(T)} & \leq \sum_{n \in \mathcal{N}_{T} \backslash \mathcal{N}_{c}}\|v\|_{L^{1}\left(\sigma_{n}\right)}\left\|\psi_{n}\right\|_{L^{\infty}\left(\sigma_{n}\right)}\left\|\partial_{i} \phi_{n}\right\|_{L^{2}(T)} \\
& \leq C h_{i, T}^{-1} \sum_{|\alpha| \leq 2} h_{T}^{\alpha}\left\|D^{\alpha} v\right\|_{L^{2}(T)}, \quad i=1,2 .
\end{aligned}
$$

Choosing now $v=u-I_{T} u$ and exploiting the fact that $\left[I_{T} u\right](n)=0$ if $n \in \mathcal{N}_{\mathrm{c}}$ (this implies $D_{h} I_{T} u=I_{T} u$ ) yields

$$
\begin{aligned}
\left\|\partial_{i}\left(u-D_{h}\right) u\right\|_{L^{2}(T)} & \leq C\left[\left\|\partial_{i}\left(u-I_{T} u\right)\right\|_{L^{2}(T)}+\left\|\partial_{i} D_{h}\left(u-I_{T} u\right)\right\|_{L^{2}(T)}\right] \\
& \leq C h_{i, T}^{-1} \sum_{|\alpha| \leq 2} h_{T}^{\alpha}\left\|D^{\alpha}\left(u-I_{T} u\right)\right\|_{L^{2}(T)} .
\end{aligned}
$$


On a reference element $\hat{T}$ we may apply the standard interpolation error estimate $\left\|\hat{u}-I_{T} \hat{u}\right\|_{H^{1}(\hat{T})} \leq|\hat{u}|_{H^{2}(\hat{T})}$ and we get

$$
\left\|\partial_{i}\left(u-D_{h}\right) u\right\|_{L^{2}(T)} \leq C h_{i, T}^{-1} \sum_{|\alpha|=2} h_{T}^{\alpha}\left\|D^{\alpha} u\right\|_{L^{2}(T)} \leq C \sum_{|\alpha|=1} h_{T}^{\alpha}\left|D^{\alpha} u\right|_{H^{1}(T)} .
$$

If $T$ does not contain a coupling node, then estimate (3.15) does not hold, but if the norm over $T$ is replaced by the norm over the patch $S_{T}$ on the right-hand side. Certainly, the property $D_{h} w=w$ on $T$ holds for an arbitrary $w \in P_{1}$ and the technique from the proof of Lemma 3.1 can be applied again. We then obtain

$$
\left\|\partial_{i}\left(u-D_{h} u\right)\right\|_{L^{2}(T)} \leq C h_{i, T}^{-1} \sum_{|\alpha| \leq 2} h_{T}^{\alpha}\left\|D^{\alpha}(u-w)\right\|_{L^{2}\left(S_{T}\right)} \leq C h_{i, T}^{-1} \sum_{|\alpha|=2} h_{T}^{\alpha}\left\|D^{\alpha} u\right\|_{L^{2}(T)}
$$

by again using the Deny-Lions type argument (3.13) with $\ell=2$ and $m=0$. With $h_{i, T} \simeq \max \left\{h_{j, T}, j=1,2,3\right\}$ we get the assertion.

It remains to prove interpolation error estimates for needle elements such that we will assume $h_{1, T}=h_{2, T} \leq$ $C h_{3, T}$ for the next lemmas. Those elements never contain interior coupling nodes.

Lemma 3.5 (stability in direction perpendicular to singular edge, anisotropic needle element away from singular edge). Assume that the element $T$ does not contain a node $n \in \mathcal{N}_{\mathrm{S}}$ and that $h_{1, T}=h_{2, T} \leq C h_{3, T}$. Then for $i=1,2$ we have

$$
\left\|\partial_{i} D_{h} u\right\|_{L^{2}(T)} \leq C\left(|u|_{H^{1}\left(S_{T}\right)}+h_{3, T}\left|\partial_{3} u\right|_{H^{1}\left(S_{T}\right)}\right)
$$

provided that $u \in H^{1}\left(S_{T}\right)$ and $\partial_{3} u \in H^{1}\left(S_{T}\right)$.

Proof. For each node $n \in \mathcal{N}_{T}$ we denote by $F_{n, T}$ the top or bottom face of the prismatic domain $S_{T}$ such that $n \in \bar{F}_{n, T}$. Observe that we have $\sigma_{n} \subset \bar{F}_{n, T} \subset \bar{S}_{T}$ for all $n \in \mathcal{N}_{T}$. Observe further that $F_{n, T}$ is isotropic with diameter of order $h_{1, T}$ and recall the standard trace inequality

$$
\|v\|_{L^{1}\left(\sigma_{n}\right)} \leq C\left|\sigma_{n}\right|\left|F_{n, T}\right|^{-1}\left(\|v\|_{L^{1}\left(F_{n, T}\right)}+h_{1, T}|v|_{W^{1,1}\left(F_{n, T}\right)}\right)
$$

for all $v \in W^{1,1}\left(F_{n, T}\right)$. We need also the trace inequality

$$
\|v\|_{L^{1}\left(F_{n, T}\right)} \leq C\left|F_{n, T} \| S_{T}\right|^{-1}\left(\|v\|_{L^{1}\left(S_{T}\right)}+h_{3, T}\left\|\partial_{3} v\right\|_{L^{1}\left(S_{T}\right)}\right)
$$

which can be proved by using Lemma A.3 and the facts that $S_{T}$ is a union of prisms, and $F_{n, T}$ is a face of $S_{T}$.

Let $s_{T}$ be one of the short edges of $T$ and denote its endpoints by $n^{1}$ and $n^{2}$. We use the same notation $s_{T}$ for the direction of this edge in order to denote by $\partial_{s_{T}} v=\nabla v \cdot s_{T} /\left|s_{T}\right|$ the directional derivative. In the following we first estimate $\left\|\partial_{s_{T}} D_{h} u\right\|_{L^{2}(T)}$. After that, the desired estimates (3.16) easily follow as we will show.

Notice that if $n \in \mathcal{N}_{T} \backslash\left\{n^{1}, n^{2}\right\}$ we have $\partial_{s_{T}} \phi_{n}=0$, and if $n \in\left\{n^{1}, n^{2}\right\}$ then $\left\|\partial_{s_{T}} \phi_{n}\right\|_{L^{\infty}(T)}=\left|s_{T}\right|^{-1} \leq C h_{1, T}^{-1}$. For all $w \in P_{0}\left(S_{T}\right)$ we have (and here we use that the element does not contain a node $n \in \mathcal{N}_{\mathrm{c}} \cup \mathcal{N}_{\mathrm{s}}$ )

$$
\begin{aligned}
\left\|\partial_{s_{T}} D_{h} u\right\|_{L^{2}(T)} & =\left\|\partial_{s_{T}} D_{h}(u-w)\right\|_{L^{2}(T)} \\
& \leq \sum_{n \in \mathcal{N}_{T} \cap s_{T}}\left|\int_{\sigma_{n}}(u-w) \psi_{n}\right|\left\|\partial_{s_{T}} \phi_{n}\right\|_{L^{2}(T)} \\
& \leq C h_{1, T}^{-1}|T|^{1 / 2} \sum_{n \in \mathcal{N}_{T} \cap s_{T}}\|u-w\|_{L^{1}\left(\sigma_{n}\right)}\left\|\psi_{n}\right\|_{L^{\infty}\left(\sigma_{n}\right)} \\
& \leq C h_{1, T}^{-1}|T|^{1 / 2} \sum_{n \in \mathcal{N}_{T} \cap s_{T}}\left|\sigma_{n}\right|^{-1}\|u-w\|_{L^{1}\left(\sigma_{n}\right)} .
\end{aligned}
$$


From the trace inequality (3.17) we have for each $n \in \mathcal{N}_{T} \cap s_{T}$

$$
\|u-w\|_{L^{1}\left(\sigma_{n}\right)} \leq C\left|\sigma_{n}\right|\left|F_{n, T}\right|^{-1}\left(\|u-w\|_{L^{1}\left(F_{n, T}\right)}+h_{1, T}|u|_{W^{1,1}\left(F_{n, T}\right)}\right) .
$$

Since the definition of $F_{n, T}$ implies $F_{n^{1}, T}=F_{n^{2}, T}=: F_{T}$, we have

$$
\|u-w\|_{L^{1}\left(\sigma_{n}\right)} \leq C\left|\sigma_{n} \| F_{T}\right|^{-1}\left(\|u-w\|_{L^{1}\left(F_{T}\right)}+h_{1, T}|u|_{W^{1,1}\left(F_{T}\right)}\right) .
$$

Now we choose $w$ as the average of $u$ on $F_{T}$ and use a Poincaré type inequality on $F_{T}$ to get

$$
\|u-w\|_{L^{1}\left(\sigma_{n}\right)} \leq C\left|\sigma_{n}\right|\left|F_{T}\right|^{-1} h_{1, T}|u|_{W^{1,1}\left(F_{T}\right)} .
$$

Therefore we arrive at

$$
\begin{aligned}
\left\|\partial_{s_{T}} D_{h} u\right\|_{L^{2}(T)} & \leq C|T|^{1 / 2}\left|F_{T}\right|^{-1}|u|_{W^{1,1}\left(F_{T}\right)} \\
& \leq C|T|^{1 / 2}\left|S_{T}\right|^{-1}\left(|u|_{W^{1,1}\left(S_{T}\right)}+h_{3, T}\left|\partial_{3} u\right|_{W^{1,1}\left(S_{T}\right)}\right) \\
& \leq C\left|S_{T}\right|^{-1 / 2}\left(|u|_{W^{1,1}\left(S_{T}\right)}+h_{3, T}\left|\partial_{3} u\right|_{W^{1,1}\left(S_{T}\right)}\right) \\
& \leq C\left(|u|_{H^{1}\left(S_{T}\right)}+h_{3, T}\left|\partial_{3} u\right|_{H^{1}\left(S_{T}\right)}\right)
\end{aligned}
$$

where we used again the trace inequality (3.18).

Now, let $s_{1, T}$ and $s_{2, T}$ be two different short edges (edge vectors) of $T$ such that the determinant of the matrix made up of $\frac{s_{1, T}}{\left|s_{1, T}\right|}, \frac{s_{2, T}}{\left|s_{2, T}\right|}$ and $\mathbf{e}_{\mathbf{3}}$ as columns is greater than a constant depending only the maximum angle of $T$. Note that this is possible due to the maximal angle condition, see [18]. Then, if the canonical vector $\mathrm{e}_{i}, i=1,2$, is expressed as

$$
\mathrm{e}_{i}=c_{1, i} \frac{s_{1, T}}{\left|s_{1, T}\right|}+c_{2, i} \frac{s_{2, T}}{\left|s_{2, T}\right|}+c_{3, i} \mathrm{e}_{3},
$$

it follows that $c_{1, i}, c_{2, i}$ and $c_{3, i}$ are bounded by above by a constant depending only on the maximum angle condition. Since

$$
\partial_{i}=c_{1, i} \partial_{s_{1, T}}+c_{2, i} \partial_{s_{2, T}}+c_{3, i} \partial_{3}
$$

we obtain (3.16) from (3.20) with $s_{T}=s_{1, T}$ and $s_{T}=s_{2, T}$, Lemma 3.3, and recalling that $h_{1, T}=h_{2, T} \leq$ $C h_{3, T}$.

Lemma 3.6 (stability in direction perpendicular to singular edge, anisotropic needle element at the singular edge, full regularity). Assume that $T \in \mathcal{T}_{h}$ is an element belonging to the macro-element $\Lambda_{\ell}$ touching the singular edge $e_{\ell}$ of that macro-element. Define the extended patch

$$
\tilde{S}_{T}:=\bigcup\left\{S_{T^{\prime}}: \mathcal{N}_{T} \cap \mathcal{N}_{\mathrm{s}} \cap \mathcal{N}_{T^{\prime}} \neq \emptyset, T^{\prime} \subset \Lambda_{j} \text { with } e_{\ell} \subset \Lambda_{j} \cap \Lambda_{\ell}\right\},
$$

$\left(\Lambda_{j}\right.$ denotes a macro-element) which may intersect multiple macro-elements. Then for $i=1,2$ the stability estimate

$$
\left\|\partial_{i} D_{h} u\right\|_{L^{2}(T)} \leq C\left(|u|_{H^{1}\left(\tilde{S}_{T}\right)}+h_{3, T}\left|\partial_{3} u\right|_{H^{1}\left(\tilde{S}_{T}\right)}\right)
$$

holds, provided that $u \in H^{2}\left(\tilde{S}_{T}\right) \cap H_{0}^{1}(\Omega)$.

Proof. As pointed out in the proof of Lemma 3.5, it is enough to estimate $\partial_{s_{T}}$ for two different short edges $s_{T}$ of $T$. For nodes on the singular edge, $n \in \mathcal{N}_{T} \cap \mathcal{N}_{\mathrm{s}}$, we define a corresponding $\sigma_{n}$ as a short edge with $n \in \overline{\sigma_{n}}$ and $\sigma_{n} \subset \partial \Omega$ (note that we did not define $\sigma_{n}$ for $n \in \mathcal{N}_{\mathrm{s}}$ on p. 1122). If $n \in \mathcal{N}_{T} \cap \mathcal{N}_{\mathrm{s}}$ is a singular vertex (belonging to a singular edge) then we further assume that $\sigma_{n}$ is a short edge of some element belonging to a 


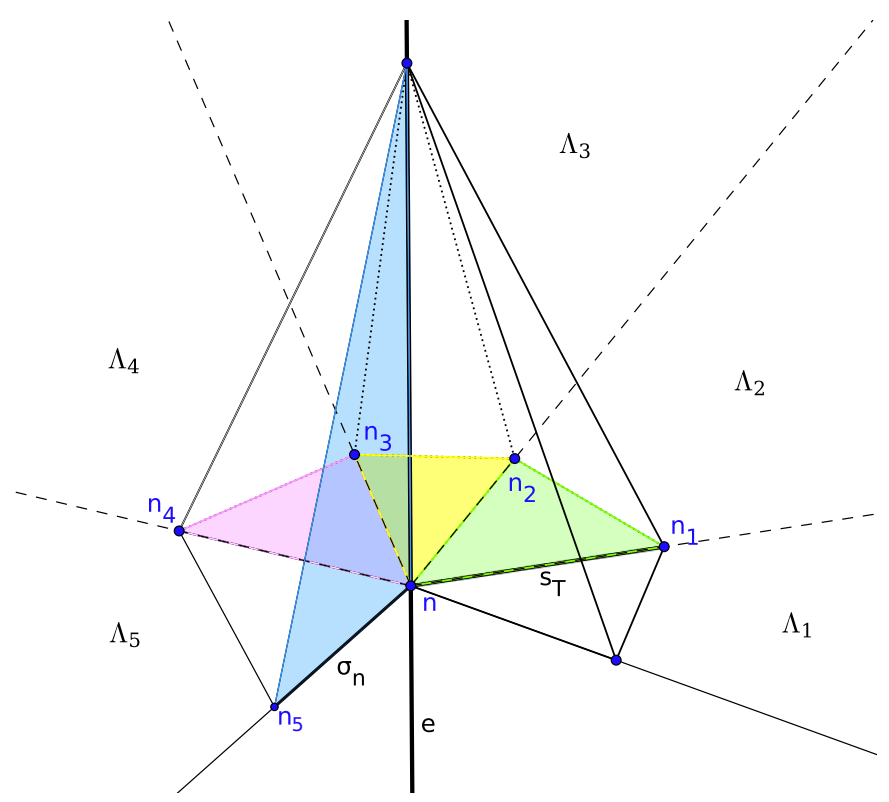

Figure 4. Possible situation where $\sigma_{n}$ for $n \in \mathcal{N}_{\mathrm{s}}$ belongs to a different macro-element than $T$.

Edge $\sigma_{n}$ is on the boundary.

macro-element that shares the singular edge $e_{\ell}$ with the macro-element $\Lambda_{\ell}$ containing $T$. In any case, we have $\sigma_{n} \subset \overline{\tilde{S}_{T}}$. Throughout the proof we write

$$
\tilde{D}_{h} u:=D_{h} u+\sum_{n \in \mathcal{N}_{T} \cap \mathcal{N}_{\mathrm{s}}}\left(\Pi_{\sigma_{n}} u\right) \cdot \varphi_{n} .
$$

We point out that $\tilde{D}_{h}$ may change with $T$. Let $s_{T}$ denote a short edge having endpoints $n \in \mathcal{N}_{\mathrm{s}}$ and $n_{1} \in \mathcal{N}_{T} \backslash \mathcal{N}_{\mathrm{s}}$. Note that Lemma 3.5 would hold for the interpolant $\tilde{D}_{h}$ if $\sigma_{n}$ and $\sigma_{n_{1}}$ would belong to the same macro-element. Since this is not always the case in the present situation we show that we can reduce it to the case where Lemma 3.5 is applicable. For simplification we consider the special case depicted in Figure 4, and one easily confirms that the following technique holds also for more general cases. We may decompose the derivative along $s_{T}$ as follows:

$$
\begin{aligned}
\left\|\partial_{s_{T}} \tilde{D}_{h} u\right\|_{L^{2}(T)} & =\left|s_{T}\right|^{-1}\left\|\tilde{D}_{h} u\left(n_{1}\right)-\tilde{D}_{h} u(n)\right\|_{L^{2}(T)} \\
& =\left|s_{T}\right|^{-1}|T|^{1 / 2}\left|\tilde{D}_{h} u\left(n_{1}\right)-\tilde{D}_{h} u(n)\right| \\
& \leq C\left|s_{T}\right|^{-1}\left(\sum_{i=1}^{3}\left|T_{i+1}\right|^{1 / 2}\left|\tilde{D}_{h} u\left(n_{i}\right)-\tilde{D}_{h} u\left(n_{i+1}\right)\right|+\left|T_{5}\right|^{1 / 2}\left|\tilde{D}_{h} u\left(n_{4}\right)-\tilde{D}_{h} u(n)\right|\right) \\
& \leq C\left(\sum_{i=1}^{3}\left\|\partial_{n_{i} n_{i+1}} \tilde{D}_{h} u\right\|_{L^{2}\left(T_{i+1}\right)}+\left\|\partial_{n n_{4}} \tilde{D}_{h} u\right\|_{L^{2}\left(T_{5}\right)}\right)
\end{aligned}
$$

where $T_{i+1}$ is an element having $n, n_{i}$ and $n_{i+1}$ as nodes and contained in the macroelent $\Lambda_{i+1}$. We remark that, if $n$ and $m$ are nodes of an edge $s$, we denote by $\partial_{n m}$ a derivative in the direction of $s$. Since $\sigma_{n_{i}}$ and $\sigma_{n_{i+1}}$ (resp. $\sigma_{4}$ and $\sigma_{n}$ ) belong to the same macro-element $\Lambda_{i+1}$ (resp. $\Lambda_{5}$ ) we obtain using the same arguments as in the proof of Lemma 3.5

$$
\left\|\partial_{n_{i} n_{i+1}} \tilde{D}_{h} u\right\|_{L^{2}\left(T_{i+1}\right)} \leq C\left(|u|_{H^{1}\left(\tilde{S}_{T}\right)}+h_{3, T}\left|\partial_{3} u\right|_{H^{1}\left(\tilde{S}_{T}\right)}\right) .
$$


Analogously, this estimate also follows for $\left\|\partial_{n n_{4}} \tilde{D}_{h} u\right\|_{L^{2}\left(T_{5}\right)}$. Inserting (3.23) into (3.22) leads to the desired estimate for $\tilde{D}_{h}$. Due to $u \in H_{0}^{1}(\Omega)$ we have $\tilde{D}_{h} u=D_{h} u$ and the proof is finished.

Lemma 3.7 (Stability in direction perpendicular to singular edge, anisotropic needle element at the singular edge). Assume that the element $T$ contains at least one node $n \in \mathcal{N}_{\mathrm{s}}$ and that $h_{1, T}=h_{2, T} \leq C h_{3, T}$. Then we have for $i=1,2$

$$
\left\|\partial_{i} D_{h} u\right\|_{L^{2}(T)} \leq C\left|S_{T}\right|^{-1 / 2}\left(|u|_{W^{1,1}\left(S_{T}\right)}+\frac{h_{3, T}}{h_{i, T}}\left\|\partial_{3} u\right\|_{L^{1}\left(S_{T}\right)}+\sum_{|\alpha|=1} h_{T}^{\alpha}\left|D^{\alpha} u\right|_{W^{1,1}\left(S_{T}\right)}\right)
$$

provided that $u \in W^{2,1}\left(S_{T}\right)$.

Proof. For each node $n \in \mathcal{N}_{\mathrm{s}} \cap \mathcal{N}_{T}$ we select one short edge $\sigma_{n}$ with an endpoint at $n$ and contained in the same macro-element as $T$ such that we can apply estimate (3.20) from the proof of Lemma 3.5 (note that we did not define $\sigma_{n}$ for $n \in \mathcal{N}_{\mathrm{s}}$ on p. 1122). We have for $i=1,2$

$$
\left\|\partial_{i}\left(D_{h} u+\sum_{n \in \mathcal{N}_{\mathrm{s}} \cap \mathcal{N}_{T}}\left(\Pi_{\sigma_{n}} u\right)(n) \phi_{n}\right)\right\|_{L^{2}(T)} \leq C\left|S_{T}\right|^{-1 / 2}\left(|u|_{W^{1,1}\left(S_{T}\right)}+h_{3, T}\left|\partial_{3} u\right|_{W^{1,1}\left(S_{T}\right)}\right) .
$$

Now we deal with $\left\|\partial_{i}\left[\left(\Pi_{\sigma_{n}} u\right)(n) \phi_{n}\right]\right\|_{L^{2}(T)}$ which is first estimated by

$$
\left\|\partial_{i}\left[\left(\Pi_{\sigma_{n}} u\right)(n) \phi_{n}\right]\right\|_{L^{2}(T)} \leq C\left\|\partial_{i} \phi_{n}\right\|_{L^{2}(T)}\left|\sigma_{n}\right|^{-1}\|u\|_{L^{1}\left(\sigma_{n}\right)}
$$

for each $n \in \mathcal{N}_{\mathrm{s}} \cap \mathcal{N}_{T}$.

Let $n \in \mathcal{N}_{\mathrm{S}} \cap \mathcal{N}_{T}$ and be $F_{n, T}$ be the face of $S_{T}$ having $\sigma_{n}$ as an edge and another edge on the singular edge. Let $P_{n, T}$ be the greatest parallelogram contained in $F_{n, T}$ and having $\sigma_{n}$ as an edge. So, $P_{n, T}$ is parallel to the $x_{3}$-axis, and its area is comparable with the area of $F_{n, T}$ since opposite edges of the trapezoid $F_{n, T}$ have equivalent length. Using a trace inequality we have

$$
\|u\|_{L^{1}\left(\sigma_{n}\right)} \leq C\left|\sigma_{n} \| F_{n, T}\right|^{-1}\left(\|u\|_{L^{1}\left(P_{n, T}\right)}+h_{3, T}\left\|\partial_{3} u\right\|_{L^{1}\left(P_{n, T}\right)}\right) .
$$

But, since $u=0$ on the edge of $P_{n, T}$ contained on the singular edge we can use the Poincaré inequality to obtain

$$
\|u\|_{L^{1}\left(\sigma_{n}\right)} \leq C\left|\sigma_{n} \| F_{n, T}\right|^{-1}\left(\left|\sigma_{n}\right|\left\|\partial_{\sigma_{n}} u\right\|_{L^{1}\left(P_{n, T}\right)}+h_{3, T}\left\|\partial_{3} u\right\|_{L^{1}\left(P_{n, T}\right)}\right) .
$$

From Lemma A.4 we have for all $v \in W^{1,1}\left(S_{T}\right)$

$$
\|v\|_{L^{1}\left(P_{n, T}\right)} \leq C\left|F_{n, T}\right|\left|S_{T}\right|^{-1}\left(\|v\|_{L^{1}\left(S_{T}\right)}+\left|s_{1, T}\right|\left\|\partial_{s_{1, T}} v\right\|_{L^{1}\left(S_{T}\right)}+\left|s_{2, T}\right|\left\|\partial_{s_{2, T}} v\right\|_{L^{1}\left(S_{T}\right)}\right) .
$$

Using twice (3.28) we obtain from (3.27)

$$
\begin{aligned}
\|u\|_{L^{1}\left(\sigma_{n}\right)} \leq & C\left|\sigma_{n}\right|^{2}\left|S_{T}\right|^{-1}\left(\left\|\partial_{\sigma_{n}} u\right\|_{L^{1}\left(S_{T}\right)}+\left|s_{1, T}\right|\left\|\partial_{s_{1, T}} \partial_{\sigma_{n}} u\right\|_{L^{1}\left(S_{T}\right)}+\left|s_{2, T}\right|\left\|\partial_{s_{2, T}} \partial_{\sigma_{n}} u\right\|_{L^{1}\left(S_{T}\right)}\right) \\
& +C\left|\sigma_{n}\right|\left|S_{T}\right|^{-1} h_{3, T}\left(\left\|\partial_{3} u\right\|_{L^{1}\left(S_{T}\right)}+\left|s_{1, T}\right|\left\|\partial_{s_{1, T}} \partial_{3} u\right\|_{L^{1}\left(S_{T}\right)}+\left|s_{2, T}\right|\left\|\partial_{s_{2, T}} \partial_{3} u\right\|_{L^{1}\left(S_{T}\right)}\right) .
\end{aligned}
$$

With the estimates

$$
\begin{aligned}
\left\|\partial_{\sigma_{n}} u\right\|_{L^{1}\left(S_{T}\right)} & \leq|u|_{W^{1,1}\left(S_{T}\right)}, \\
\left\|\partial_{s_{i, T}} \partial_{\sigma_{n}} u\right\|_{L^{1}\left(S_{T}\right)} & \leq|u|_{W^{2,1}\left(S_{T}\right)}, \quad i=1,2, \\
\left\|\partial_{s_{i, T}} \partial_{3} u\right\|_{L^{1}\left(S_{T}\right)} & \leq\left|\partial_{3} u\right|_{W^{1,1}\left(S_{T}\right)}, \quad i=1,2,
\end{aligned}
$$


the inequality

$$
\left\|\partial_{i} \phi_{n}\right\|_{L^{2}(T)} \leq C h_{i, T}^{-1}|T|^{1 / 2},
$$

and $\left|\sigma_{n}\right| \sim h_{i, T}(i=1,2)$ we obtain from (3.26)

$$
\begin{aligned}
\left\|\partial_{i}\left[\left(\Pi_{\sigma_{n}} u\right)(n) \phi_{n}\right]\right\|_{L^{2}(T)} \leq & C\left|S_{T}\right|^{-1 / 2}\left(|u|_{W^{1,1}\left(S_{T}\right)}+\left(h_{1, T}+h_{2, T}\right)|u|_{W^{2,1}\left(S_{T}\right)}\right) \\
& +C\left|S_{T}\right|^{-1 / 2}\left(\frac{h_{3, T}}{h_{i, T}}\left\|\partial_{3} u\right\|_{L^{1}\left(S_{T}\right)}+h_{3, T}\left|\partial_{3} u\right|_{W^{1,1}\left(S_{T}\right)}\right) .
\end{aligned}
$$

Finally, taking into account that, since $h_{1, T}=h_{2, T} \leq C h_{3, T}$, we have

$$
\left(h_{1, T}+h_{2, T}\right)|u|_{W^{2,1}\left(S_{T}\right)}+h_{3, T}\left|\partial_{3} u\right|_{W^{1,1}\left(S_{T}\right)} \leq C \sum_{|\alpha|=1} h_{T}^{\alpha}\left|D^{\alpha} u\right|_{W^{1,1}\left(S_{T}\right)},
$$

inequality (3.24) follows from (3.25) and (3.30).

We are now prepared to estimate the interpolation error for needle elements.

Lemma 3.8 (anisotropic needle element, full regularity). If $T$ is an anisotropic element with $h_{1, T}=h_{2, T} \leq$ $C h_{3, T}$ and $\mathcal{N}_{\mathrm{s}} \cap \mathcal{N}_{T}=\emptyset$, then the local interpolation error estimate

$$
\left|u-D_{h} u\right|_{H^{1}(T)} \leq C \sum_{|\alpha|=1} h_{T}^{\alpha}\left|D^{\alpha} u\right|_{H^{1}\left(S_{T}\right)}
$$

holds provided that $u \in H^{2}\left(S_{T}\right)$. Assume $T$ touches the singular edge, that is $\mathcal{N}_{\mathbf{s}} \cap \mathcal{N}_{T} \neq \emptyset$. Let $\tilde{S}_{T}$ be the patch defined in Lemma 3.6, then the estimate

$$
\left|u-D_{h} u\right|_{H^{1}(T)} \leq C \sum_{|\alpha|=1} h_{T}^{\alpha}\left|D^{\alpha} u\right|_{H^{1}\left(\tilde{S}_{T}\right)}
$$

holds, if $u \in H^{2}\left(\tilde{S}_{T}\right) \cap H_{0}^{1}(\Omega)$.

\section{Remark 3.9.}

1. The estimate (3.31) does not hold for the Lagrange interpolant, see [2].

2. This lemma will be applied in the proof of Theorem 3.11 in two situations: for the singular part of the solution of the boundary value problem and away from the edge, but also for the regular part of the solution for all $T$.

Proof of Lemma 3.8. We have full regularity and in case that $T$ does not touch the singular edge, we may apply the stability estimates of Lemmas 3.3 and 3.5 which leads to

$$
\begin{aligned}
\left|D_{h} u\right|_{H^{1}(T)} & \leq C \sum_{|\alpha| \leq 1} h_{T}^{\alpha}\left\|D^{\alpha} \partial_{3} u\right\|_{L^{2}\left(S_{T}\right)}+C\left(|u|_{H^{1}\left(S_{T}\right)}+h_{3, T}\left|\partial_{3} u\right|_{H^{1}\left(S_{T}\right)}\right) \\
& \leq C \sum_{|\alpha| \leq 1} h_{T}^{\alpha}\left|D^{\alpha} u\right|_{H^{1}\left(S_{T}\right)} .
\end{aligned}
$$

We exploit that $D_{h} w=w$ for an arbitrary $w \in \mathcal{P}_{1}$. Consequently, we get

$$
\begin{aligned}
\left|u-D_{h} u\right|_{H^{1}(T)} & =\left|(u-w)-D_{h}(u-w)\right|_{H^{1}(T)} \\
& \leq|u-w|_{H^{1}(T)}+\left|D_{h}(u-w)\right|_{H^{1}(T)} \\
& \leq C \sum_{|\alpha| \leq 1} h_{T}^{\alpha}\left|D^{\alpha}(u-w)\right|_{H^{1}\left(S_{T}\right)} .
\end{aligned}
$$


We use now again the Deny-Lions type argument (3.13) with $\ell=2$ and $m=1$, and conclude the desired estimate.

For the case that $T$ touches the singular edge we use the modified interpolant (3.21) and replace $S_{T}$ by $\tilde{S}_{T}$ in the above considerations. Moreover, the stability estimate of Lemma 3.6 has to be used then in the first step of this proof. With $\tilde{D}_{h} u=D_{h} u$ for $u$ vanishing on the boundary we get the desired estimate.

Lemma 3.10 (anisotropic needle element, reduced regularity). Let $T$ be an anisotropic element with $h_{1, T}=$ $h_{2, T} \leq C h_{3, T}$ and let $S_{T}$ have zero distance to the singular edge. Then the local interpolation error estimate

$$
\left|u-D_{h} u\right|_{H^{1}(T)} \leq C h_{1, T}^{1-\delta} \sum_{i=1}^{2}\left\|\partial_{i} u\right\|_{V_{\delta, \delta}^{1,2}\left(S_{T}\right)}+C h_{3, T}\left\|\partial_{3} u\right\|_{V_{0,0}^{1,2}\left(S_{T}\right)}
$$

holds provided that $u$ has the regularity demanded by the right-hand sides of the estimates and $\delta \in[0,1)$. If $T$ is an element with $h_{1, T}=h_{2, T} \leq C h_{3, T}$ and $S_{T}$ has zero distance to both a singular vertex and a singular edge then the local interpolation error estimate

$$
\left|u-D_{h} u\right|_{H^{1}(T)} \leq C h_{1, T}^{1-\beta-\delta} h_{3, T}^{\delta} \sum_{i=1}^{2}\left\|\partial_{i} u\right\|_{V_{\beta, \delta}^{1,2}\left(S_{T}\right)}+C h_{1, T}^{-\beta} h_{3, T}\left\|\partial_{3} u\right\|_{V_{\beta, 0}^{1,2}\left(S_{T}\right)}
$$

hold provided that $u$ has the regularity demanded by the right-hand sides of the estimates and $\beta, \delta \in[0,1)$, $\beta+\delta<1$.

Proof. As in the proof of Lemma 3.8 we distinguish between the derivatives $\partial_{3} D_{h} u$ and the derivatives along directions perpendicular to the $x_{3}$-axis. From Lemma 3.3 we obtain by using the triangle inequality and $\left|S_{T}\right|^{-1 / 2}\left\|\partial_{3} u\right\|_{L^{1}\left(S_{T}\right)} \leq\left\|\partial_{3} u\right\|_{L^{2}(T)}$

$$
\begin{aligned}
\left\|\partial_{3}\left(u-D_{h} u\right)\right\|_{L^{2}(T)} & \leq\left\|\partial_{3} u\right\|_{L^{2}(T)}+C\left|S_{T}\right|^{-1 / 2} \sum_{|\alpha| \leq 1} h_{T}^{\alpha}\left\|D^{\alpha} \partial_{3} u\right\|_{L^{1}\left(S_{T}\right)} \\
& \leq C\left\|\partial_{3} u\right\|_{L^{2}\left(S_{T}\right)}+C\left|S_{T}\right|^{-1 / 2} \sum_{|\alpha|=1} h_{T}^{\alpha}\left\|D^{\alpha} \partial_{3} u\right\|_{L^{1}\left(S_{T}\right)} .
\end{aligned}
$$

For the estimate of $\partial_{i} D_{h} u, i=1,2$, we use Lemma 3.7, from which we conclude that

$$
\left\|\partial_{i}\left(u-D_{h} u\right)\right\|_{L^{2}(T)} \leq C|u|_{H^{1}\left(S_{T}\right)}+C\left|S_{T}\right|^{-1 / 2}\left(\frac{h_{3, T}}{h_{i, T}}\left\|\partial_{3} u\right\|_{L^{1}\left(S_{T}\right)}+\sum_{|\alpha|=1} h_{T}^{\alpha}\left|D^{\alpha} u\right|_{W^{1,1}\left(S_{T}\right)}\right) .
$$

These two estimates can be summarized to

$$
\begin{aligned}
& \left|u-D_{h} u\right|_{H^{1}(T)} \\
& \leq C|u|_{H^{1}\left(S_{T}\right)}+C\left|S_{T}\right|^{-1 / 2}\left(\frac{h_{3, T}}{h_{1, T}}\left\|\partial_{3} u\right\|_{L^{1}\left(S_{T}\right)}+\sum_{|\alpha|=1} h_{T}^{\alpha}\left|D^{\alpha} u\right|_{W^{1,1}\left(S_{T}\right)}\right) .
\end{aligned}
$$

It remains to estimate the terms against the weighted norms. In case that $S_{T}$ touches the singular vertex we have

$$
\begin{aligned}
|u|_{H^{1}\left(S_{T}\right)} & \leq \sum_{i=1}^{2}\left\|R^{1-\beta} \theta^{1-\delta} \cdot R^{\beta-1} \theta^{\delta-1} \partial_{i} u\right\|_{L^{2}(T)}+\left\|R^{1-\beta} \theta \cdot R^{\beta-1} \theta^{-1} \partial_{3} u\right\|_{L^{2}(T)} \\
& \leq \sum_{i=1}^{2} \max _{S_{T}} R^{1-\beta} \theta^{1-\delta}\left\|\partial_{i} u\right\|_{V_{\beta, \delta}^{1,2}\left(S_{T}\right)}+\max _{S_{T}} R^{1-\beta}\left\|\partial_{3} u\right\|_{V_{\beta, 0}^{1,2}\left(S_{T}\right)} .
\end{aligned}
$$


With $R^{1-\beta} \theta^{1-\delta}=r^{1-\delta} R^{-\beta} R^{\delta} \leq r^{1-\beta-\delta} R^{\delta} \leq C h_{1, T}^{1-\beta-\delta} h_{3, T}^{\delta}$ (where we used the assumption $\beta+\delta \leq 1$ ) and $R^{1-\beta} \leq h_{3, T}^{1-\beta} \leq h_{1, T}^{-\beta} h_{3, T}$ we derive

$$
|u|_{H^{1}\left(S_{T}\right)} \leq C h_{1, T}^{1-\beta-\delta} h_{3, T}^{\delta} \sum_{i=1}^{2}\left\|\partial_{i} u\right\|_{V_{\beta, \delta}^{1,2}\left(S_{T}\right)}+C h_{1, T}^{-\beta} h_{3, T}\left\|\partial_{3} u\right\|_{V_{\beta, 0}^{1,2}\left(S_{T}\right)} .
$$

With $R^{1-\delta} \theta^{1-\delta}=r^{1-\delta} \leq C h_{1, T}^{1-\delta}$ (using that the exponent is positive) we derive also

$$
|u|_{H^{1}\left(S_{T}\right)} \leq C h_{1, T}^{1-\delta} \sum_{i=1}^{2}\left\|\partial_{i} u\right\|_{V_{\delta, \delta}^{1,2}\left(S_{T}\right)}+C h_{3, T}\left\|\partial_{3} u\right\|_{V_{0,0}^{1,2}\left(S_{T}\right)} .
$$

In the other case that $T$ intersects the singular edge, but no singular vertex, we have

$$
\frac{h_{3, T}}{h_{1, T}}\left\|\partial_{3} u\right\|_{L^{1}\left(S_{T}\right)} \leq \frac{h_{3, T}}{h_{1, T}}\left\|\partial_{3} u\right\|_{V_{0,0}^{1,2}\left(S_{T}\right)}\|r\|_{L^{2}\left(S_{T}\right)} \leq h_{3, T}\left|S_{T}\right|^{1 / 2}\left\|\partial_{3} u\right\|_{V_{0,0}^{1,2}\left(S_{T}\right)} .
$$

If $T$ has also a singular vertex, then we have with $R^{\beta-1} \theta^{-1}=R^{\beta} r^{-1}$

$$
\frac{h_{3, T}}{h_{1, T}}\left\|\partial_{3} u\right\|_{L^{1}\left(S_{T}\right)} \leq \frac{h_{3, T}}{h_{1, T}}\left\|\partial_{3} u\right\|_{V_{\beta, 0}^{1,2}\left(S_{T}\right)}\left\|R^{-\beta} r\right\|_{L^{2}\left(S_{T}\right)} \leq h_{3, T} h_{1, T}^{-\beta}\left|S_{T}\right|^{1 / 2}\left\|\partial_{3} u\right\|_{V_{\beta, 0}^{1,2}\left(S_{T}\right)}
$$

where we used that

$$
\left\|R^{-\beta} r\right\|_{L^{2}\left(S_{T}\right)} \leq\left\|r^{1-\beta}\right\|_{L^{2}\left(S_{T}\right)} \leq C h_{1, T}^{1-\beta}\left|S_{T}\right|^{1 / 2}
$$

which can be obtained by integration. The second derivatives in estimate (3.34) are treated in a similar way. For $i=1,2,3$ we get

$$
\left\|\partial_{i 3} u\right\|_{L^{1}\left(S_{T}\right)} \leq\left\|R^{-\beta}\right\|_{L^{2}\left(S_{T}\right)}\left\|R^{\beta} \partial_{i 3} u\right\|_{L^{2}\left(S_{T}\right)} \leq h_{1, T}^{-\beta}\left|S_{T}\right|^{1 / 2}\left\|\partial_{3} u\right\|_{V_{\beta, 0}^{1,2}\left(S_{T}\right)} .
$$

For $i, j=1,2$ and supposing that $T$ does not have singular vertices we have

$$
\begin{aligned}
h_{i, T}\left\|\partial_{i j} u\right\|_{L^{1}\left(S_{T}\right)} & \leq h_{1, T}\left\|R^{-\delta} \theta^{-\delta}\right\|_{L^{2}\left(S_{T}\right)}\left\|R^{\delta} \theta^{\delta} \partial_{i j} u\right\|_{L^{2}\left(S_{T}\right)} \\
& \leq h_{1, T}^{1-\delta}\left|S_{T}\right|^{1 / 2}\left|\partial_{i} u\right|_{V_{\delta, \delta}^{1,2}\left(S_{T}\right)}
\end{aligned}
$$

where we used again an argument as in (3.35). If $T$ has a singular vertex, then

$$
h_{i, T}\left\|\partial_{i j} u\right\|_{L^{1}\left(S_{T}\right)} \leq h_{1, T}\left\|R^{-\beta} \theta^{-\delta}\right\|_{L^{2}\left(S_{T}\right)}\left\|R^{\beta} \theta^{\delta} \partial_{i j} u\right\|_{L^{2}\left(S_{T}\right)} .
$$

Note that $R^{-\beta} \theta^{-\delta}=R^{-\beta+\delta} r^{-\delta} \leq R^{\delta} r^{-\beta-\delta} \leq h_{3, T}^{\delta} r^{-\beta-\delta}$ hold due to $\beta+\delta<1$. The norm $\left\|r^{-\beta-\delta}\right\|_{L^{2}\left(S_{T}\right)}$ can be computed exactly by executing the integration which leads to

$$
\left\|R^{-\beta} \theta^{-\delta}\right\|_{L^{2}\left(S_{T}\right)} \leq h_{3, T}^{\delta}\left\|r^{-\beta-\delta}\right\|_{L^{2}\left(S_{T}\right)} \leq C h_{1, T}^{-\beta-\delta} h_{3, T}^{\delta}\left|S_{T}\right|^{1 / 2} .
$$

Hence we have

$$
h_{i, T}\left\|\partial_{i j} u\right\|_{L^{1}\left(S_{T}\right)} \leq C h_{1, T}^{1-\beta-\delta} h_{3, T}^{\delta}\left|S_{T}\right|^{1 / 2}\left\|\partial_{i} u\right\|_{V_{\beta, \delta}^{1,2}\left(S_{T}\right)} .
$$

Therefore, the desired estimates are proved. 
Theorem 3.11 (global interpolation error estimate). Let $u$ be the solution of the boundary value problem (1.1) with $f \in L^{2}(\Omega)$, and let $u_{I}, u_{R}$ be the functions obtained from the splitting (2.2). Assume that the refinement parameters $\mu_{\ell}$ and $\nu_{\ell}$ satisfy the conditions

$$
\begin{aligned}
\mu_{\ell} & <\lambda_{\mathrm{e}}^{(\ell)}, \\
\nu_{\ell} & <\lambda_{\mathrm{v}}^{(\ell)}+\frac{1}{2}, \\
\frac{1}{\nu_{\ell}}+\frac{1}{\mu_{\ell}}\left(\lambda_{\mathrm{v}}^{(\ell)}-\frac{1}{2}\right) & >1
\end{aligned}
$$

$\ell=1, \ldots, L$. Then the global interpolation error estimate

$$
\left|u_{R}-D_{h} u_{R}\right|_{H^{1}\left(\Lambda_{\ell}\right)} \leq C h\|f\|_{L^{2}(\Omega)}
$$

is satisfied.

Proof. The estimation of the global error can be reduced to the evaluation of the global error to each macroelement $\Lambda_{\ell}$. So we will consider such a subdomain $\Lambda_{\ell}$ with one singular edge and one singular vertex. The other cases are treated in an even simpler way. We will omit the index $\ell$.

In view of Theorem 2.1 and the decompositions (2.1) and (2.2) we have $u_{R}=\left(u_{\mathrm{r}}-u_{I}\right)+u_{\mathrm{s}}$ with $u_{\mathrm{r}}-u_{I} \in$ $H^{2}(\Lambda)$, so we need to consider the interpolation error for both the regular part, which we denote now by $w:=u_{\mathrm{r}}-u_{I}$, and the singular part $u_{\mathrm{s}}$. Note, that the choice of $u_{s}$ is not unique and it is possible to guarantee the property $u_{s}(n)=0$ for all $n \in \mathcal{N}_{\mathrm{c}}$. This implies $w(n)=0$ for $n \in \mathcal{N}_{\mathrm{c}}$ which is used later.

We begin with an estimate for the regular part $w$. Since

$$
\left|w-D_{h} w\right|_{H^{1}(\Lambda)}^{2}=\sum_{T \subset \Lambda}\left|w-D_{h} w\right|_{H^{1}(T)}^{2}
$$

we can use the local interpolation estimates from Lemma 3.1 when $T$ is isotropic, Lemma 3.4 when $T$ is flat, and Lemma 3.8 when $T$ is a needle element away from the singular edge, which yields

$$
\left|w-D_{h} w\right|_{H^{1}(T)} \leq C h_{T}|w|_{H^{2}\left(S_{T}\right)}=h_{T}\left|u_{r}\right|_{H^{2}\left(S_{T}\right)}
$$

for all these cases. For those $T$ which touch the singular edge the local estimate of Lemma 3.8 reads now

$$
\left|w-D_{h} w\right|_{H^{1}(T)}=\left|u_{r}-D_{h} u_{r}\right|_{H^{1}(T)} \leq C h_{T}\left|u_{r}\right|_{H^{2}\left(\tilde{S}_{T}\right)} .
$$

Here, we have a patch $\tilde{S}_{T}$ on the right-hand side which may be contained in multiple macro-elements. Inserting now (3.41) and (3.42) into (3.40) yields

$$
\left|w-D_{h} w\right|_{H^{1}(\Lambda)}^{2} \leq C h^{2}\left|u_{r}\right|_{H^{2}(\Omega)}^{2} \leq C h^{2}\|f\|_{L^{2}(\Omega)}^{2},
$$

where we used that $h_{T}(=\operatorname{diam}(T)) \leq h$ for all $T \in \mathcal{T}_{h}$ and that the $S_{T}$ and $\tilde{S}_{T}$ may overlap only a finite number (independent of $h$ ) of times.

Now consider the estimate of $\left|u_{\mathrm{s}}-D_{h} u_{\mathrm{s}}\right|_{H^{1}(\Lambda)}$. Firstly, denote by $\mathrm{T}_{R}$ the set of all elements $T \subset \Lambda$ whose patches $S_{T}$ are away from the singular edge and from the singular vertex. This also implies

$$
R_{T} \geq h^{1 / \nu}, \quad r_{T} \geq h^{1 / \mu} .
$$

We further set $\Lambda_{R}=\cup\left\{T: T \in \mathrm{T}_{R}\right\}$. Since $u_{s} \in H^{2}\left(S_{T}\right)$ on these elements we use Lemmas 3.1, 3.4 or 3.8 as appropriate to have

$$
\left|u_{\mathrm{s}}-D_{h} u_{\mathrm{s}}\right|_{H^{1}(T)} \leq \sum_{|\alpha|=1} h_{T}^{\alpha}\left|D^{\alpha} u_{\mathrm{s}}\right|_{H^{1}\left(S_{T}\right)} .
$$


Due to the mesh condition we have $h_{i, T} \leq C h r_{T}^{1-\mu} \leq h r(x)^{1-\mu}$ for $i=1,2$ and $h_{3, T} \leq C h R_{T}^{1-\nu} \leq h R(x)^{1-\nu}$ for all $x \in S_{T}$ and thus the estimates

$$
\begin{aligned}
h_{i, T}\left|\partial_{i} u_{\mathrm{s}}\right|_{H^{1}\left(S_{T}\right)} & \leq h\left|u_{\mathrm{s}}\right|_{V_{1-\mu, 1-\mu}^{1,2}\left(S_{T}\right)}, \quad \text { for } i=1,2, \text { and } \\
h_{3, T}\left|\partial_{3} u_{\mathrm{s}}\right|_{H^{1}\left(S_{T}\right)} & \leq h\left|u_{\mathrm{s}}\right|_{V_{1-\nu, 0}^{1,2}\left(S_{T}\right)}
\end{aligned}
$$

follow. Notice that due to our assumptions upon $\mu$ and $\nu$ the inequalities

$$
1-\mu>1-\lambda_{\mathrm{e}}, \quad 1-\nu>\frac{1}{2}-\lambda_{\mathrm{v}}, \quad \text { and } \quad 1-\mu>\frac{1}{2}-\lambda_{\mathrm{v}}
$$

hold, where the last estimate is equivalent to

$$
\frac{1}{\mu}+\frac{1}{\mu}\left(\lambda_{\mathrm{v}}-\frac{1}{2}\right)>1
$$

which follows from (3.38) taking $\mu \leq \nu$ into account. Due to the estimates (3.44) Theorem 2.1 can be applied and we obtain

$$
|u|_{V_{1-\mu, 1-\mu}^{1,2}(\Lambda)} \leq C\|f\|_{L^{2}(\Omega)}, \quad|u|_{V_{1-\nu, 0}^{1,2}(\Lambda)} \leq C\|f\|_{L^{2}(\Omega)} .
$$

Then summing up all the elements $T \in \mathrm{T}_{R}$ we arrive at

$$
\left|u_{\mathrm{s}}-D_{h} u_{\mathrm{s}}\right|_{H^{1}\left(\Lambda_{R}\right)} \leq C h\|f\|_{L^{2}(\Omega)} .
$$

Secondly, let $\mathrm{T}_{E}$ be the set of elements $T$ which are away from the singular vertex, i.e. $R_{T} \geq h^{1 / \nu}$, but such that $\overline{S_{T}}$ intersects the singular edge, and set $\Lambda_{E}=\cup\left\{T: T \in \mathrm{T}_{E}\right\}$. We use inequality (3.32) of Lemma 3.10 (which is valid when $T$ is isotropic too) with $\delta=1-\mu$. Due to $h_{3, T} \leq C h R_{T}^{1-\nu}$ on $S_{T}$, we get

$$
h_{3, T}\left\|\partial_{3} u_{\mathrm{s}}\right\|_{V_{0,0}^{1,2}\left(S_{T}\right)} \leq h\left\|\partial_{3} u_{\mathrm{s}}\right\|_{V_{1-\nu, 0}^{1,2}\left(S_{T}\right)} .
$$

As in the previous case we now sum up all elements $T \in T_{E}$ and apply Theorem 2.1 exploiting the properties (3.44) again. This leads to

$$
\begin{aligned}
\left\|\partial_{i} u_{\mathrm{s}}\right\|_{V_{1-\mu, 1-\mu}^{1,2}\left(\Lambda_{E}\right)} & \leq C\|f\|_{L^{2}(\Omega)}, \quad i=1,2, \\
\left\|\partial_{3} u_{\mathrm{s}}\right\|_{V_{1-\nu, 0}^{1,2}\left(\Lambda_{E}\right)} & \leq C\|f\|_{L^{2}(\Omega)},
\end{aligned}
$$

and as a consequence we obtain

$$
\left|u_{\mathrm{s}}-D_{h} u_{\mathrm{s}}\right|_{H^{1}\left(\Lambda_{E}\right)} \leq C h\|f\|_{L^{2}(\Omega)} .
$$

Finally, we denote by $\mathrm{T}_{S}$ the set of all the elements $T$ such that $\overline{S_{T}}$ contains the singular vertex, and by $\Lambda_{S}$ the union of all such elements. If $T$ is one of these elements, we have $h_{1, T}=h_{2, T} \leq C h^{1 / \mu}$ and $h_{3, T} \leq C h^{1 / \nu}$. So using inequality (3.33) of Lemma 3.10, we obtain

$$
\left|u_{\mathrm{s}}-D_{h} u_{\mathrm{s}}\right|_{H^{1}\left(\Lambda_{S}\right)} \leq C h^{\frac{1-\beta-\delta}{\mu}+\frac{\delta}{\nu}}\left(\left\|\partial_{1} u_{\mathrm{s}}\right\|_{V_{\beta, \delta}^{1,2}(\Lambda)}+\left\|\partial_{2} u_{\mathrm{s}}\right\|_{V_{\beta, \delta}^{1,2}(\Lambda)}\right)+C h^{\frac{1}{\nu}-\frac{\beta}{\mu}}\left\|\partial_{3} u_{\mathrm{s}}\right\|_{V_{\beta, 0}^{1,2}(\Lambda)}
$$

for $\beta, \delta \in[0,1)$ with $\beta+\delta<1$. Let us now construct feasible weights $\beta$ and $\delta$ which satisfy the assumptions of Theorem 2.1 and yield the expected convergence rate. We set $\beta=1 / 2-\lambda_{\mathrm{v}}+\varepsilon$ for some $\varepsilon>0$ which is sufficiently small such that the estimates (3.46)-(3.48) below hold. Due to $\mu \leq \nu$ and (3.37) we get

$$
\frac{1-\beta-\delta}{\mu}+\frac{\delta}{\nu} \geq \frac{1-\beta}{\nu}=\frac{1 / 2+\lambda_{\mathrm{v}}-\varepsilon}{\nu} \geq 1 .
$$


Moreover, with the definition of $\beta$ and (3.38) we get

$$
\frac{1}{\nu}-\frac{\beta}{\mu}=\frac{1}{\nu}-\frac{1}{\mu}\left(\frac{1}{2}-\lambda_{\mathrm{v}}\right)-\frac{\varepsilon}{\mu} \geq 1 .
$$

Furthermore, we have to confirm that a feasible $\delta$ exists, which means that the inequalities $\delta<1-\beta=1 / 2+\lambda_{\mathrm{v}}-\varepsilon$ and $\delta>1-\lambda_{\mathrm{e}}$ have to hold simultaneously. A suitable choice of $\delta$ is possible since $\lambda_{\mathrm{v}}>0, \lambda_{\mathrm{e}}>1 / 2$ and

$$
1 / 2<\lambda_{\mathrm{e}}+\lambda_{\mathrm{v}}-\varepsilon
$$

Inserting now (3.46) and (3.47) into (3.45) and and applying Theorem 2.1 yields

$$
\left|u_{\mathrm{S}}-D_{h} u_{\mathrm{s}}\right|_{H^{1}\left(\Lambda_{S}\right)} \leq C h\|f\|_{\Omega} .
$$

Since $\bar{\Lambda}=\bar{\Lambda}_{R} \cup \bar{\Lambda}_{E} \cup \bar{\Lambda}_{S}$ the proof is complete.

Remark 3.12. The refinement conditions (3.36)-(3.38) were discussed in [4] already: the conditions (3.36) and (3.37) balance the edge and vertex singularities. The third condition, (3.38), follows from (3.37) in the case $\mu_{\ell}=\nu_{\ell} ;$ only in the case $\mu_{\ell}<\nu_{\ell}$ it imposes a condition between $\mu_{\ell}$ and $\nu_{\ell}$ limiting the anisotropy of the mesh. For the Fichera example treated in Section 4 we have $\lambda_{\mathrm{v}}^{(\ell)} \approx 0.454$ and $\lambda_{\mathrm{e}}^{(\ell)}=\frac{2}{3}$. With the choice $\nu_{\ell}=0.9$ the conditions (3.36) and (3.38) imply the choice $0.414<\mu_{\ell}<\frac{2}{3}$. For $\nu_{\ell}=0.8$ we would get the weaker condition $0.184<\mu_{\ell}<\frac{2}{3}$.

Note also that in the absence of singularities we have set $\lambda_{\mathrm{e}}^{(\ell)}=\infty$ and/or $\lambda_{\mathrm{v}}^{(\ell)}=\infty$. In these cases we can set $\mu_{\ell}=1$ and/or $\nu_{\ell}=1$.

Corollary 3.13 ( $H^{1}$ and $L^{2}$ finite element error estimate). Let $u$ be the solution of the boundary value problem (1.1), and let $u_{h}$ be the corresponding finite element solution on a finite element mesh as constructed in Section 2 with grading parameters satisfying the conditions (3.36)-(3.38). Then the discretization error can be estimated by

$$
\begin{aligned}
\left\|u-u_{h}\right\|_{H^{1}(\Omega)} & \leq C h\|f\|_{L^{2}(\Omega)}, \\
\left\|u-u_{h}\right\|_{L^{2}(\Omega)} & \leq C h^{2}\|f\|_{L^{2}(\Omega)} .
\end{aligned}
$$

Proof. We choose $v_{h}=u_{I}+D_{h} u_{R}$ in estimate (1.4) and observe that $u-v_{h}=u_{R}-D_{h} u_{R}$. With Lemma 3.11 we obtain the estimate (3.49). The $L^{2}$-error estimate can be derived by the standard Aubin-Nitsche method.

Remark 3.14. A trivial conclusion from (3.49) is the stability estimate

$$
\left\|u_{h}\right\|_{H^{1}(\Omega)} \leq C\|f\|_{L^{2}(\Omega)}
$$

which we will need in Section 5.

Remark 3.15. In macro-elements of Type 4 with $\mu_{\ell}=\nu_{\ell}<1$, Apel and Nicaise suggested in [4] the use of a more elegant refinement strategy as depicted in Figure 5. Our proof cannot be transfered to this kind of mesh immediately since there may be elements $T$ where $S_{T}$ is not prismatic as it was exploited in the proof of Lemmas 3.5 and 3.7. We conjecture that the assertion still holds but do not pursue this further in this paper.

\section{Numerical test}

As in [4] we consider the Poisson problem (1.1) in the "Fichera domain" $\Omega:=(-1,1)^{3} \backslash[0,1]^{3}$ and choose the right-hand side $f=1+R^{-3 / 2} \ln ^{-1}(R / 4)$ which is in $L^{2}(\Omega)$ but not in $L^{p}(\Omega)$ for $p>2$. For this problem 


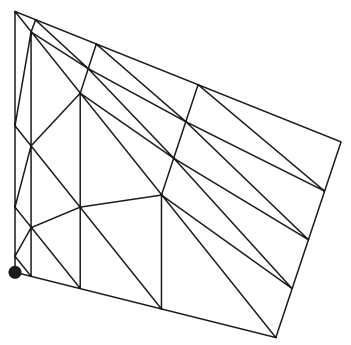

FiguRE 5. Modification of macro-element of Type 4.

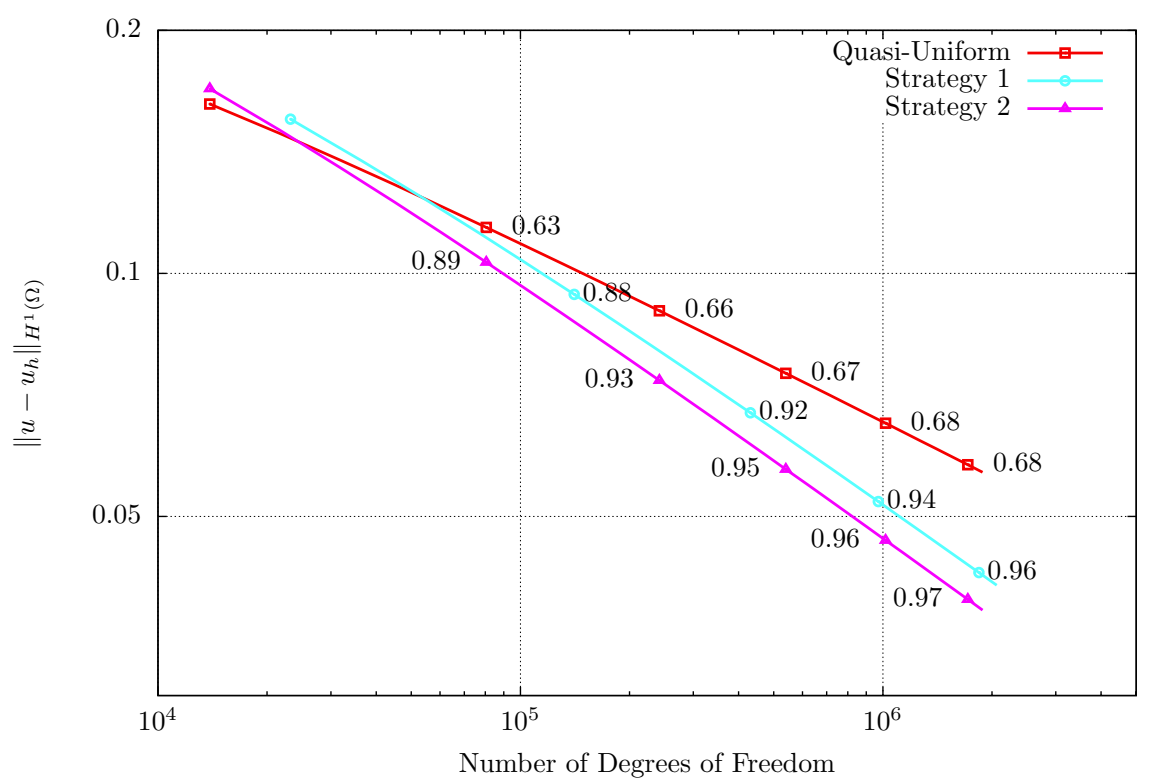

FiguRE 6. Plot of the estimated error against the number of unknowns. The labels at the curve denote the estimated convergence order in terms of $h \sim N^{-1 / 3}$. Strategy 1: anisotropic refinement using Type 1-Type 4; Strategy 2: anisotropic refinement using the modified rule of Remark 3.15 instead of Type 4 .

we have $\lambda_{v} \approx 0.45$ for the concave vertex [30] and $\lambda_{e}=\frac{\pi}{\omega_{0}}=\frac{2}{3}$ for the three concave edges. All other edges and vertices are non-singular.

This boundary value problem was solved on quasi-uniform and on graded meshes with our refinement strategy using $\mu=\nu=0.5<\min \left\{\lambda_{e}, \lambda_{v}+\frac{1}{2}\right\}$, where Type 1, Type 2 and Type 4 occur. Additionally we include the strategy where the macros of Type 4 are replaced by the modified version from Remark 3.15. Pictures of such meshes can be found in [4]. The refinement strategies and an a posteriori error estimator of residual type [32] were implemented into the finite element package MooNMD [19]. The estimated error norms are plotted against the number of unknowns in Figure 6. We see that the theoretical approximation order $h^{1} \sim N^{-1 / 3}$ from Corollary 3.13 can be verified in the practical calculation for both refinement strategies. The error with the second strategy is slightly smaller. We denoted by $N$ the number of nodes. 


\section{DiscretizATION ERROR ESTIMATES FOR A DiSTRIBUted OPTIMAL CONTROL PROBLEM}

Hinze introduced the variational discretization concept for linear-quadratic control constrained optimal control problems in [17]. We follow here this concept in a special case. Consider the minimization of

$$
J(y, u):=\frac{1}{2}\left\|y-y_{d}\right\|_{L^{2}(\Omega)}^{2}+\frac{\alpha}{2}\|u\|_{L^{2}(\Omega)}^{2},
$$

with the constraint that the state $y \in H_{0}^{1}(\Omega)$ is the weak solution of the Poisson problem

$$
-\Delta y=u \quad \text { in } \Omega, \quad y=0 \quad \text { on } \partial \Omega,
$$

and, that the control $u$ is restricted by constant bounds $u_{a}, u_{b} \in \mathbb{R}$, this means that the set of admissible controls is defined by

$$
U^{\text {ad }}:=\left\{u \in L^{2}(\Omega): u_{a} \leq u \leq u_{b} \text { a.e. } \Omega\right\} .
$$

The regularization parameter $\alpha$ is a fixed positive number and $y_{d} \in L^{2}(\Omega)$ is the desired state. It is well known that this problem has a unique optimal solution $(\bar{y}, \bar{u})$. There is an optimal adjoint state $\bar{p} \in H_{0}^{1}(\Omega)$, and the triplet $(\bar{y}, \bar{u}, \bar{p})$ satisfies the first order optimality conditions

$$
\begin{aligned}
(\nabla \bar{y}, \nabla v)_{L^{2}(\Omega)} & =(\bar{u}, v)_{L^{2}(\Omega)} & & \forall v \in H_{0}^{1}(\Omega), \\
(\nabla \bar{p}, \nabla v)_{L^{2}(\Omega)} & =\left(\bar{y}-y_{d}, v\right)_{L^{2}(\Omega)} & & \forall v \in H_{0}^{1}(\Omega), \\
(\alpha \bar{u}+\bar{p}, u-\bar{u})_{L^{2}(\Omega)} & \geq 0 & & \forall u \in U^{\mathrm{ad}} .
\end{aligned}
$$

With the variational discretization concept the approximate solution is obtained by replacing $H_{0}^{1}(\Omega)$ by a finite element space $V_{h} \subset H_{0}^{1}(\Omega)$ and searching $\left(\bar{y}_{h}, \bar{u}_{h}, \bar{p}_{h}\right) \in V_{h} \times U^{\text {ad }} \times V_{h}$ such that

$$
\begin{aligned}
\left(\nabla \bar{y}_{h}, \nabla v_{h}\right)_{L^{2}(\Omega)} & =\left(\bar{u}_{h}, v_{h}\right)_{L^{2}(\Omega)} & & \forall v_{h} \in V_{h}, \\
\left(\nabla \bar{p}_{h}, \nabla v_{h}\right)_{L^{2}(\Omega)} & =\left(\bar{y}_{h}-y_{d}, v_{h}\right)_{L^{2}(\Omega)} & & \forall v_{h} \in V_{h}, \\
\left(\alpha \bar{u}_{h}+\bar{p}_{h}, u-\bar{u}_{h}\right)_{L^{2}(\Omega)} & \geq 0 & & \forall u \in U^{\mathrm{ad}} .
\end{aligned}
$$

Note that the control space is not discretized; nevertheless $\bar{u}_{h}$ can be obtained by the projection of $-\bar{p}_{h} / \alpha$ onto $U^{\text {ad }}$, see [17]. The discretization error estimate

$$
\left\|\bar{u}-\bar{u}_{h}\right\|_{L^{2}(\Omega)}+\left\|\bar{y}-\bar{y}_{h}\right\|_{L^{2}(\Omega)}+\left\|\bar{p}-\bar{p}_{h}\right\|_{L^{2}(\Omega)} \leq C h^{2}\left(\|\bar{u}\|_{L^{2}(\Omega)}+\left\|y_{d}\right\|_{L^{2}(\Omega)}\right)
$$

can be concluded from (3.50) and (3.51), see [7,17]. With the proof of Corollary 3.13 we have established this result for anisotropic discretizations of the state equation (5.1) in the case of three-dimensional polyhedral domains.

\section{Discrete compactness Property for EDGE Elements}

The Discrete Compactness Property (DCP) is a useful tool to study the convergence of finite element discretizations of the Maxwell equations, both for eigenvalue and source problems. It was first introduced by Kikuchi [20] and proved for Nédélec edge elements of lowest order on tetrahedral shape regular meshes. We refer to the monograph by Monk [23] and the references therein for further analysis on isotropic meshes. The property was also analyzed on anisotropically refined tetrahedral meshes on polyhedra for edge elements of lowest order by Nicaise [25] (excluding vertex singularities) and by Buffa et al. [13].

Lombardi [21] extended this result to edge elements of arbitrary order, also including vertex and edge singularities. The proof is based on two tools: 1) interpolation error estimates for edge elements on meshes satisfying the maximum angle condition, and 2) interpolation error estimates for a piecewise linear interpolation operator 
defined on $W^{2, p}(\Omega) \cap H_{0}^{1}(\Omega), p \geq 2$, preserving boundary conditions. For the latter, the Lagrange interpolation was used (implying $p>2$ ) together the results of Apel and Nicaise [4], giving some artificial restrictions on the grading parameters defining the allowed anisotropically graded meshes. Using now estimate (3.49) of Corollary 3.13 we can extend the result of [21] allowing little more general meshes.

In what follows we define a family of edge element spaces and introduce the DCP for this family. We refer to [21] for further definitions and notation. First we introduce the divergence-free space

$$
X=\left\{\mathbf{v} \in H_{0}(\operatorname{curl}, \Omega): \operatorname{div} \mathbf{v}=0 \text { on } \Omega\right\} .
$$

Then we introduce discretizations of this space where the divergence-free condition is weakly imposed. Let I be a denumerable set of positive real numbers having 0 as the only limit point. From now till the end of this section, we assume that $h \in \mathrm{I}$. For each $h$, let $\mathcal{T}_{h}$ be the mesh on the polyhedron $\Omega$ constructed in Section 2 . Given an integer $k \geq 1$, let $X_{h}$ be the space defined as

$$
X_{h}=\left\{\mathbf{v}_{h} \in H_{0}(\operatorname{curl}, \Omega):\left.\mathbf{v}_{h}\right|_{T} \in \mathcal{N}_{k}(T) \forall T \in \mathcal{T}_{h},\left(\nabla p_{h}, \mathbf{v}_{h}\right)_{L^{2}(\Omega)}=0 \forall p_{h} \in S_{h}\right\}
$$

where $\mathcal{N}_{k}(T)$ is the space of edge elements of order $k$ on $T$, and

$$
S_{h}=\left\{p_{h} \in H_{0}^{1}(\Omega):\left.p_{h}\right|_{T} \in P_{k}(T) \forall T \in \mathcal{T}_{h}\right\} .
$$

We say that the family of spaces $\left\{X_{h}\right\}_{h \in \mathrm{I}}$ satisfies the discrete compactness property if for each sequence $\left\{\mathbf{v}_{h}\right\}_{h \in \mathrm{J}}, \mathrm{J} \subset \mathrm{I}$, verifying for a constant $C$

$$
\begin{aligned}
& \mathbf{v}_{h} \in X_{h}, \quad \forall h \in \mathrm{J}, \\
& \left\|\mathbf{v}_{h}\right\|_{H_{0}(\mathbf{c u r l}, \Omega)} \leq C, \quad \forall h \in \mathrm{J},
\end{aligned}
$$

there exists a function $\mathbf{v} \in X$ and a subsequence $\left\{\mathbf{v}_{h_{n}}\right\}_{n \in \mathbb{N}}$ such that (for $n \rightarrow \infty$ )

$$
\begin{array}{ll}
\mathbf{v}_{h_{n}} \rightarrow \mathbf{v} & \text { in } L^{2}(\Omega) \\
\mathbf{v}_{h_{n}} \rightarrow \mathbf{v} & \text { weakly in } H_{0}(\operatorname{curl}, \Omega) .
\end{array}
$$

Theorem 6.1. If the grading parameters defining the meshes $\mathcal{T}_{h}$ satisfy the conditions (3.36)-(3.38), then the family of spaces $\left\{X_{h}\right\}_{h>0}$ verifies the discrete compactness property.

Proof. Follow exactly the arguments used to prove Theorem 5.2 of [21] taking into account that the inequality (4.21) of that paper is now a consequence of estimate (3.49).

\section{Appendix A. Proof of trace inequalities}

We start from the known trace inequality

$$
\|u\|_{L^{p}(\hat{\sigma})} \leq \hat{C}\|u\|_{W^{1, r}(\hat{F})} \quad \forall u \in W^{1, r}(\hat{F})
$$

where $1 \leq p \leq r \leq \infty, \hat{F}$ is the reference triangle (with vertices $(0,0),(1,0)$ and $(0,1)), \hat{\sigma}$ is one of its edges and $\hat{C}$ is a constant depending only on $p$ and $r$. It follows for instance from Theorem 4.2 of [24]. If $F$ is a triangle and $\sigma$ is one of its edges, we obtain by a simple rescaling argument (we assume here $r<\infty$.)

$$
\|u\|_{L^{p}(\sigma)} \leq C|\sigma|^{1 / p}|F|^{-1 / r}\left(\|u\|_{L^{r}(F)}+\left|\ell_{1}\right|\left\|\partial_{\ell_{1}} u\right\|_{L^{r}(F)}+\left|\ell_{2}\right|\left\|\partial_{\ell_{2}} u\right\|_{L^{r}(F)}\right) \quad \forall u \in W^{1, r}(F),
$$

with the constant $C$ depending only on $p$ and $r$, and where $\ell_{1}$ and $\ell_{2}$ are two arbitrary sides of $F$. 
Similarly, if $T$ is a tetrahedron, $F$ is one of its faces, $\ell_{i}, i=1,2,3$ are three non coplanar edges of $T$, and if $1 \leq r \leq q<\infty$, we have the inequality

$$
\|u\|_{L^{r}(F)} \leq C|F|^{1 / r}|T|^{-1 / q}\left(\|u\|_{L^{q}(T)}+\sum_{i=1}^{3}\left|\ell_{i} \| \partial_{\ell_{i}} u\right|_{L^{q}(T)}\right) \quad \forall u \in W^{1, q}(T),
$$

with $C$ depending only on $r$ and $q$. In fact, (A.2) is obtained by a rescaling argument from the trace inequality

$$
\|u\|_{L^{r}(\hat{F})} \leq \hat{C}\|u\|_{W^{1, q}(\hat{T})} \quad \forall u \in W^{1, q}(\hat{T})
$$

for a reference element $\hat{T}$ with a face $\hat{F}$, taking as reference element either the tetrahedron with vertices at $(0,0,0),(1,0,0),(0,1,0)$ and $(0,0,1)$ when the edges $\ell_{i}$ are concurrent, or the tetrahedron with vertices at $(0,0,0),(1,1,0),(0,1,0)$ and $(0,0,1)$ when they are not.

We can now combine inequalities (A.1) and (A.2). Let $T$ be a tetrahedron, $\sigma$ one of its edges, and $\ell_{i}, i=1,2,3$ three non coplanar edges. Then if $1 \leq p \leq q<\infty$ there exists a constant depending only on $p$ and $q$ such that

$$
\|u\|_{L^{p}(\sigma)} \leq C|\sigma|^{1 / p}|T|^{-1 / q}\left(\|u\|_{L^{q}(T)}+\sum_{i=1}^{3}\left|\ell_{i}\right|\left\|\partial_{\ell_{i}} u\right\|_{L^{q}(T)}+\sum_{i, j=1}^{3}\left|\ell_{i}\left\|\ell_{j} \mid\right\| \partial_{\ell_{i} \ell_{j}} u \|_{L^{q}(T)}\right) \quad \forall u \in W^{2, q}(T) .\right.
$$

Clearly, inequality (A.3) can be written as

$$
\|u\|_{L^{p}(\sigma)} \leq C|\sigma|^{1 / p}|T|^{-1 / q}\left(\|u\|_{L^{q}(T)}+h_{T}|u|_{W^{1, q}(T)}+h_{T} \sum_{|\alpha|=1} h_{T}^{\alpha}\left|D^{\alpha} u\right|_{W^{1, q}(T)}\right) \quad \forall u \in W^{2, q}(T),
$$

where the constant $C$ depends on $p, q$ and the shape-regularity constant of $T$. However (A.4) is also valid on some arbitrarily anisotropic elements with a uniformly bounded constant $C$, as we state in the next Lemma.

Lemma A.1. There exists a constant $C$ depending only on $p$ and $q$, with $1 \leq p \leq q<\infty$, but independent of $h$, such that for all $T \in \mathcal{T}_{h}$ and $\sigma$ edge of $T$ the inequality (A.4) holds.

Remark A.2. In (A.4) the derivative $D^{\alpha}$ refers to a partial derivative with respect to the local Cartesian system of the macro-element in which $T$ is included.

Proof. The conclusion follows from (A.3) if we prove that there exists a constant $C$ independent of $h$ such that any tetrahedron $T \in \mathcal{T}_{h}$ has three non coplanar edges $\ell_{i}, i=1,2,3$ with

$$
\left|\ell_{i}\right| \leq C h_{i, T}, \quad\left|\ell_{i} \cdot e_{j}\right| \leq C h_{j, T}, \quad i, j=1,2,3 .
$$

In the spirit of Remark A.2, $e_{j}$ refers to the unitary vectors of the local cartesian system of the macro-elements containing $T$. This condition is clear on elements contained on isotropic refinement macro-elements (of Type 1 and Type 2). So we need to consider elements contained on macro-elements of Type 3 or Type 4. Let $T$ be one of those elements. We know that $T$ has a vertical edge $\ell_{3}$, and has two edges $\ell_{1}$ and $\ell_{2}$ contained on one or two planes transversal to the singular edge of the macro-element (introduced after proof of Thm. 2.1). Then $\ell_{1}, \ell_{2}$ and $\ell_{3}$ verify (A.5). This is a consequence of the results of [4] (see inequation (3.2)).

We also need trace inequalities where in the right hand side the presence of some derivatives is avoided. We have the following results. 


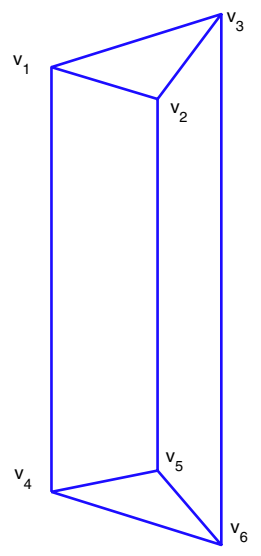

FiguRE 7. Illustration of the prism.

Lemma A.3. Let $P$ be a triangular prism with vertices $v_{i}, i=1, \ldots, 6$, where the face $v_{1} v_{2} v_{3}$ is opposite to the face $v_{4} v_{5} v_{6}$, and where the edges $v_{1} v_{4}, v_{2} v_{5}$, and $v_{3} v_{6}$ are parallel to the $x_{3}$-axis, see Figure 7 . Denote by $F$ the face $v_{1} v_{2} v_{3}$. Then for all $v \in W^{1, p}(P), p \in[1, \infty)$, we have

$$
\|v\|_{L^{p}(F)}^{p} \leq \frac{C_{\mathrm{reg}}}{\cos \gamma} \cdot h_{3}^{-1}\left(\|v\|_{L^{p}(P)}^{p}+h_{3}^{p}\left\|\partial_{3} v\right\|_{L^{p}(P)}^{p}\right)
$$

where $h_{3}$ is length of the shortest vertical edge, and $\gamma$ is the angle between the $x_{1} x_{2}$-plane and the plane containing the face $F$. The constant $C_{\text {reg }}$ depends only on $p$ and the minimum angle of the face $F$.

Proof. We can assume $v_{1}=(0,0,0)$ and $v_{4}=\left(0,0, h_{3}\right)$. Suppose $v_{2}=\left(a_{2}, b_{2}, c_{2}\right), v_{3}=\left(a_{3}, b_{3}, c_{3}\right)$. Let $s, t$ such that

$$
\begin{aligned}
& a_{2} s+b_{2} t=c_{2} \\
& a_{3} s+b_{3} t=c_{3} .
\end{aligned}
$$

It is clear that there exist such $s$ and $t$ since $v_{1}, v_{2}$, and $v_{3}$ do not lay on one line. Then the map $f(\tilde{x})=B \tilde{x}$ with

$$
B=\left(\begin{array}{lll}
1 & 0 & 0 \\
0 & 1 & 0 \\
s & t & 1
\end{array}\right)
$$

sends $\widetilde{P}$ to $P$ where $\widetilde{P}$ is a prism with three vertical edges and some of its vertices are $\tilde{v}_{1}=(0,0,0), \tilde{v}_{2}=$ $\left(a_{2}, b_{2}, 0\right), \tilde{v}_{3}=\left(a_{3}, b_{3}, 0\right)$ and $\tilde{v}_{4}=\left(0,0, h_{3}\right)$. Let $\widetilde{F}$ be the face $\tilde{v}_{1} \tilde{v}_{2} \tilde{v}_{3}$ of $\widetilde{P}$.

Let $\tilde{v}$ be defined by $\tilde{v}(\tilde{x})=v(x)$ if $x=B \tilde{x}$. Then we have

$$
\|v\|_{L^{p}(F)}^{p}=\frac{1}{\cos \gamma}\|\tilde{v}\|_{L^{p}(\widetilde{F})}^{p}
$$

Now, if $\widetilde{Q}$ is the right prism with vertices $\tilde{v}_{1}, \ldots, \tilde{v}_{4},\left(a_{2}, b_{2}, h_{3}\right)$ and $\left(a_{3}, v_{3}, h_{3}\right)$, then we have using a trace inequality on $\widetilde{Q}$ and noting that $\widetilde{Q} \subset \widetilde{P}$ that

$$
\begin{aligned}
\|\tilde{v}\|_{L^{p}(\widetilde{F})}^{p} & \leq C_{p} h_{3}^{-1}\left(\|\tilde{v}\|_{L^{p}(\widetilde{Q})}^{p}+h_{3}^{p}\left\|\tilde{\partial}_{3} \tilde{v}\right\|_{L^{p}(\widetilde{Q})}^{p}\right) \\
& \leq C_{p} h_{3}^{-1}\left(\|\tilde{v}\|_{L^{p}(\widetilde{P})}^{p}+h_{3}^{p}\left\|\tilde{\partial}_{3} \tilde{v}\right\|_{L^{p}(\widetilde{P})}^{p}\right)
\end{aligned}
$$




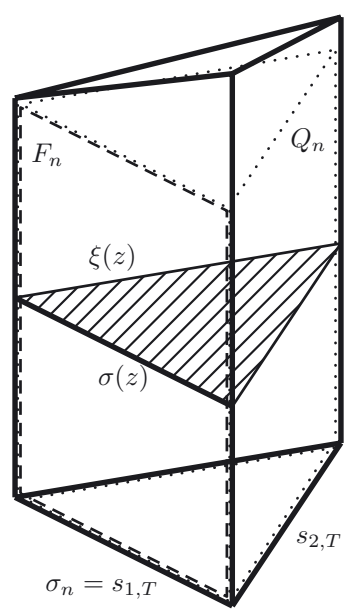

Figure 8. Illustration of the notation used in Lemma A.4. The dotted lines indicate the prism $Q_{n}$, dashed lines the parallelogram $P_{n}$ while the triangle $\xi(z)$ is hatched. Note that $\sigma(z)=\overline{\xi(z)} \cap F_{n}$.

with $C_{p}$ depending only on $p$. Therefore, we have

$$
\begin{aligned}
\|v\|_{L^{p}(F)}^{p} & =\frac{C_{p}}{\cos \gamma} h_{3}^{-1}\left(\|\tilde{v}\|_{L^{p}(\widetilde{P})}^{p}+h_{3}^{p}\left\|\tilde{\partial}_{3} \tilde{v}\right\|_{L^{p}(\tilde{P})}^{p}\right) \\
& =\frac{C_{\operatorname{reg}}}{\cos \gamma} h_{3}^{-1}|B|\left(\|v\|_{L^{p}(P)}^{p}+h_{3}^{p}\left\|\partial_{3} v\right\|_{L^{p}(P)}^{p}\right)
\end{aligned}
$$

where we used that $\tilde{\partial}_{3} \tilde{v}(\tilde{x})=\partial_{3} v(x)$. Since $|B|=1$ we obtain the desired result.

Lemma A.4. Let $T$ be an anisotropic element with the node $n$ on the singular edge and let $\sigma_{n}$ be a short edge. Let $P_{n} \subset \overline{S_{T}}$ be a parallelogram of maximal area having $\sigma_{n}$ as an edge and another edge on the singular edge, see Figure 8. And let $F_{n}$ the face of $S_{T}$ containing $P_{n}$. Then $\left|P_{n}\right| \geq C\left|F_{n}\right|$, and for all $v \in W^{1,1}\left(S_{T}\right)$ we have

$$
\|v\|_{L^{1}\left(P_{n}\right)} \leq C\left|F_{n} \| S_{T}\right|^{-1}\left(\|v\|_{L^{1}\left(S_{T}\right)}+\left|s_{1, T}\right|\left\|\partial_{s_{1, T}} v\right\|_{L^{1}\left(S_{T}\right)}+\left|s_{2, T}\right|\left\|\partial_{s_{2, T}} v\right\|_{L^{1}\left(S_{T}\right)}\right) .
$$

where $s_{1, T}$ and $s_{2, T}$ are two short edges of $T$.

Proof. The inequality $\left|P_{n}\right| \geq C\left|F_{n}\right|$ follows from our assumptions on the mesh, in particular from the comparable length of opposite edges of $F_{n}$. For proving the estimate choose the coordinate system such that $n=(0,0,0)$.

Assume first $v$ is regular. We have

$$
\begin{aligned}
\|v\|_{L^{1}\left(P_{n}\right)} & \leq C \int_{0}^{h_{3, P_{n}}} \int_{0}^{\left|\sigma_{n}\right|}\left|v\left((0,0, z)+t \sigma_{n}\right)\right| \mathrm{d} t \mathrm{~d} z \\
& =\int_{0}^{h_{3, P_{n}}} \int_{\sigma(z)}|v| \mathrm{d} s \mathrm{~d} z
\end{aligned}
$$

where $\sigma(z)$ is the segment parallel to $\sigma_{n}$ and with the same length and passing through $(0,0, z)$. If $\xi(z)$ is the triangle contained in $S_{T}$ having $\sigma(z)$ as an edge and being parallel to the bottom face of $S_{T}$, then since we can 
assume $\left.v\right|_{\xi(z)}$ is regular (because $v$ is), by the trace inequality (A.1) we have

$$
\int_{\sigma(z)}|v| \leq C \frac{\left|\sigma_{n}\right|}{|\xi|} \int_{\xi(z)}\left(|v|+\left|s_{1, T}\right|\left|\partial_{s_{1, T}} v\right|+\left|s_{2, T}\right|\left|\partial_{s_{2, T}} v\right|\right)
$$

where $\left|s_{1, T}\right|$ and $\left|s_{2, T}\right|$ are the lengths of two small edges of $T$ and $|\xi|=|\xi(0)|$. So we have

$$
\begin{aligned}
\|v\|_{L^{1}\left(P_{n}\right)} & \leq C \frac{\left|\sigma_{n}\right|}{|\xi|} \int_{0}^{h_{3, P_{n}}} \int_{\xi(z)}\left(|v|+\left|s_{1, T}\right|\left|\partial_{s_{1, T}} v\right|+\left|s_{2, T}\right|\left|\partial_{s_{2, T}} v\right|\right) \\
& \leq C \frac{\left|P_{n}\right|}{\left|Q_{n}\right|} \int_{0}^{h_{3, P_{n}}} \int_{\xi(z)}\left(|v|+\left|s_{1, T}\right|\left|\partial_{s_{1, T}} v\right|+\left|s_{2, T}\right|\left|\partial_{s_{2, T}} v\right|\right) \\
& \leq C \frac{\left|P_{n}\right|}{\left|Q_{n}\right|} \int_{Q_{n}}\left(|v|+\left|s_{1, T}\right|\left|\partial_{s_{1, T}} v\right|+\left|s_{2, T}\right|\left|\partial_{s_{2, T}} v\right|\right) \\
& \leq C \frac{\left|P_{n}\right|}{\left|Q_{n}\right|} \int_{S_{T}}\left(|v|+\left|s_{1, T}\right|\left|\partial_{s_{1, T}} v\right|+\left|s_{2, T}\right|\left|\partial_{s_{2, T}} v\right|\right)
\end{aligned}
$$

where $Q_{n}$ is the prism formed by the union of $\xi(z)$ with $z \in\left[0, h_{3, P_{n}}\right]$ that is contained in $S_{T}$. Since $\left|P_{n}\right| \leq\left|F_{n}\right|$ and, from the assumptions on the mesh, $\left|Q_{n}\right| \geq C\left|S_{T}\right|$ we arrive at

$$
\|v\|_{L^{1}\left(P_{n}\right)} \leq C \frac{\left|F_{n}\right|}{\left|S_{T}\right|} \int_{S_{T}}\left(|v|+\left|s_{1, T}\right|\left|\partial_{s_{1, T}} v\right|+\left|s_{2, T} \| \partial_{s_{2, T}} v\right|\right) .
$$

If $v \in W^{1,1}\left(S_{T}\right)$, let $\left\{v_{k}\right\}_{k}$ be a sequence of $C^{\infty}$ functions converging to $v$ in $W^{1,1}\left(S_{T}\right)$. For each $k$ we have

$$
\left\|v_{k}\right\|_{L^{1}\left(P_{n}\right)} \leq C\left|F_{n} \| S_{T}\right|^{-1}\left(\left\|v_{k}\right\|_{L^{1}\left(S_{T}\right)}+\left|s_{1, T}\right|\left\|\partial_{s_{1, T}} v_{k}\right\|_{L^{1}\left(S_{T}\right)}+\left|s_{2, T}\right|\left\|\partial_{s_{2, T}} v_{k}\right\|_{L^{1}\left(S_{T}\right)}\right) .
$$

Now, the proof concludes by taking limit as $k \rightarrow \infty$.

Acknowledgements. The authors would like to thank the anonymous referees for reading the paper carefully and giving valuable hints to improve the paper. The work of all authors was supported by DFG (German Research Foundation), IGDK 1754. The work of the second author is also supported by ANPCyT (grant PICT 2010-1675 and PICTO 2008-00089) and by CONICET (grant PIP 11220090100625). This support is gratefuly acknowledged.

\section{REFERENCES}

[1] Th. Apel, Interpolation of non-smooth functions on anisotropic finite element meshes. ESAIM: M2AN 33 (1999) 1149-1185.

[2] Th. Apel and M. Dobrowolski, Anisotropic interpolation with applications to the finite element method. Computing 47 (1992) $277-293$.

[3] Th. Apel and B. Heinrich, Mesh refinement and windowing near edges for some elliptic problem. SIAM J. Numer. Anal. 31 (1994) 695-708.

[4] Th. Apel and S. Nicaise, The finite element method with anisotropic mesh grading for elliptic problems in domains with corners and edges. Math. Methods Appl. Sci. 21 (1998) 519-549.

[5] Th. Apel, A.-M. Sändig, and J.R. Whiteman, Graded mesh refinement and error estimates for finite element solutions of elliptic boundary value problems in non-smooth domains. Math. Methods Appl. Sci. 19 (1996) 63-85.

[6] Th. Apel and D. Sirch, $L^{2}$-error estimates for Dirichlet and Neumann problems on anisotropic finite element meshes. Appl. Math. 56 (2011) 177-206.

[7] Th. Apel and D. Sirch, A priori mesh grading for distributed optimal control problems, in Constrained Optimization and Optimal Control for Partial Differential Equations, vol. 160. Edited by G. Leugering, S. Engell, A. Griewank, M. Hinze, R. Rannacher, V. Schulz, M. Ulbrich, and S. Ulbrich. Int. Ser. Numer. Math.. Springer, Basel (2011) 377-389.

[8] F. Assous, P. Ciarlet, Jr. and J. Segré, Numerical solution to the time-dependent Maxwell equations in two-dimensional singular domains: the Singular Complement Method. J. Comput. Phys. 161 (2000) 218-249.

[9] I. Babuška, Finite element method for domains with corners. Computing 6 (1970) 264-273. 
[10] A.E. Beagles and J.R. Whiteman, Finite element treatment of boundary singularities by augmentation with non-exact singular functions. Numer. Methods Partial Differ. Eqs. 2 (1986) 113-121.

[11] H. Blum and M. Dobrowolski, On finite element methods for elliptic equations on domains with corners. Computing 28 (1982) $53-63$.

[12] C. Băcuţă, V. Nistor and L.T. Zikatanov, Improving the rate of convergence of high-order finite elements in polyhedra II: mesh refinements and interpolation. Numer. Funct. Anal. Optim. 28 (2007) 775-824.

[13] A. Buffa, M. Costabel and M. Dauge, Algebraic convergence for anisotropic edge elements in polyhedral domains. Numer. Math. 101 (2005) 29-65.

[14] P. Clément, Approximation by finite element functions using local regularization. RAIRO Anal. Numer. 2 (1975) 77-84.

[15] T. Dupont and R. Scott, Polynomial approximation of functions in Sobolev spaces. Math. Comput. 34 (1980) $441-463$.

[16] P. Grisvard, Singularities in boundary value problems, vol. 22. Research Notes Appl. Math. Springer, New York (1992).

[17] M. Hinze. A variational discretization concept in control constrained optimization: The linear-quadratic case. Comput. Optim. Appl. 30 (2005) 45-61.

[18] P. Jamet, Estimations d'erreur pour des éléments finis droits presque dégénérés. R.A.I.R.O. Anal. Numer. 10 (1976) 43-61.

[19] V. John and G. Matthies, MooNMD-a program package based on mapped finite element methods. Comput. Visual. Sci. 6 (2004) 163-169.

[20] F. Kikuchi, On a discrete compactness property for the nédélec finite elements. J. Fac. Sci. Univ. Tokyo Sect. IA Math. 36 (1989) 479-490.

[21] A.L. Lombardi, The discrete compactness property for anisotropic edge elements on polyhedral domains. ESAIM: M2AN 47 (2013) 169-181.

[22] J. M.-S. Lubuma and S. Nicaise, Dirichlet problems in polyhedral domains II: approximation by FEM and BEM. J. Comput. Appl. Math. 61 (1995) 13-27,.

[23] P. Monk, Finite Element Methods for Maxwell's Equations. Oxford University Press, New York (2003).

[24] J. Nečas, Les méthodes directes en théorie des équations elliptiques. Masson et Cie, Éditeurs, Paris, Academia, Éditeurs, Paris, Prague (1967).

[25] S. Nicaise, Edge elements on anisotropic meshes and approximation of the Maxwell equations. SIAM J. Numer. Anal. 39 (2001) 784-816.

[26] L.A. Oganesyan and L.A. Rukhovets, Variational-difference schemes for linear second-order elliptic equations in a twodimensional region with piecewise smooth boundary. Zh. Vychisl. Mat. Mat. Fiz. 8 (1968) 97-114. In Russian. English translation in USSR Comput. Math. and Math. Phys. 8 (1968) 129-152.

[27] T. von Petersdorff and E.P. Stephan. Regularity of mixed boundary value problems in $\mathbb{R}^{3}$ and boundary element methods on graded meshes. Math. Methods Appl. Sci. 12 (1990) 229-249.

[28] G. Raugel, Résolution numérique de problèmes elliptiques dans des domaines avec coins. Ph.D. thesis. Université de Rennes (1978).

[29] A.H. Schatz and L.B. Wahlbin, Maximum norm estimates in the finite element method on plane polygonal domains. Part 2: Refinements. Math. Comput. 33 (1979) 465-492.

[30] H. Schmitz, K. Volk and W.L. Wendland, On three-dimensional singularities of elastic fields near vertices. Numer. Methods Partial Differ. Eqs. 9 (1993) 323-337.

[31] L.R. Scott and S. Zhang, Finite element interpolation of non-smooth functions satisfying boundary conditions. Math. Comput. 54 (1990) 483-493.

[32] K. Siebert, An a posteriori error estimator for anisotropic refinement. Numer. Math. 73 (1996) 373-398.

[33] G. Strang and G. Fix, An analysis of the finite element method. Prentice-Hall, Englewood Cliffs, NJ (1973). 